

\title{
On the arithmetic and geometry of binary Hamiltonian forms
}

\author{
Jouni Parkkonen and Frédéric Paulin \\ Appendix by Vincent Emery
}

\begin{abstract}
Given an indefinite binary quaternionic Hermitian form $f$ with coefficients in a maximal order of a definite quaternion algebra over $\mathbb{Q}$, we give a precise asymptotic equivalent to the number of nonequivalent representations, satisfying some congruence properties, of the rational integers with absolute value at most $s$ by $f$, as $s$ tends to $+\infty$. We compute the volumes of hyperbolic 5-manifolds constructed by quaternions using Eisenstein series. In the appendix, V. Emery computes these volumes using Prasad's general formula. We use hyperbolic geometry in dimension 5 to describe the reduction theory of both definite and indefinite binary quaternionic Hermitian forms.
\end{abstract}

\section{Introduction}

Following [Weyl 1940; 1942], we will call a Hermitian form over Hamilton's real quaternion algebra with anti-involution the conjugation a Hamiltonian form.

Since Gauss, the reduction theory of the integral binary quadratic forms and the problem of representation of integers by them is quite completely understood. For binary Hermitian forms, these subjects have been well studied, starting with Hermite, Bianchi and especially Humbert, and much developed by Elstrodt, Grunewald and Mennicke; see for instance [Elstrodt et al. 1998]. In the recent paper [Parkkonen and Paulin 2011], we gave a precise asymptotic on the number of nonequivalent proper representations of rational integers with absolute value at most $s$ by a given integral indefinite Hermitian form. Besides the general results on quadratic forms (see for instance [Weyl 1940; Cassels 1978]) and some special work (see for instance [Pronin 1967; Hashimoto and Ibukiyama 1980]), not much seemed to be precisely known on these questions for binary Hamiltonian forms.

The work in the appendix is supported by the Swiss National Science Foundation, project number PP00P2-128309/1.

MSC2010: primary 11E39, 11R52, 20G20; secondary 11N45, 15A21, 53A35, 11F06, 20H10.

Keywords: binary Hamiltonian form, representation of integers, group of automorphs,

Hamilton-Bianchi group, hyperbolic volume, reduction theory. 
In this paper, we use hyperbolic geometry in dimension 5 to study the asymptotic of the counting of representations of rational integers by integral binary Hamiltonian forms and to give a geometric description of the reduction theory of such forms. General formulas are known (by Siegel's mass formula; see for instance [Eskin et al. 1991]), but it does not seem to be easy (or even doable) to deduce our asymptotic formulas from them. There are numerous results on the counting of integer points with bounded norm on quadrics (or homogeneous varieties); see for instance the work of Duke, Eskin, McMullen, Oh, Rudnick, Sarnak and others. In this paper, we count appropriate orbits of integer points on which a fixed integral binary Hamiltonian form is constant, analogously to [Parkkonen and Paulin 2011].

Let $\mathbb{H}$ be Hamilton's quaternion algebra over $\mathbb{R}$, with $x \mapsto \bar{x}$ its conjugation, $\mathrm{n}: x \mapsto x \bar{x}$ its reduced norm and tr $: x \mapsto x+\bar{x}$ its reduced trace. Let $A$ be a quaternion algebra over $\mathbb{Q}$ that is definite $\left(A \otimes_{\mathbb{Q}} \mathbb{R}=\mathbb{U}\right)$, with reduced discriminant $D_{A}$ and class number $h_{A}$. Let $\mathcal{O}$ be a maximal order in $A$, and let $\mathfrak{m}$ be a (nonzero) left fractional ideal of $\mathcal{O}$, with reduced norm $\mathrm{n}(\mathfrak{m})$; see Section 2 for definitions.

Let $f: \mathbb{W} \times \mathbb{U} \rightarrow \mathbb{R}$ be a binary Hamiltonian form, with

$$
f(u, v)=a \mathrm{n}(u)+\operatorname{tr}(\bar{u} b v)+c \mathrm{n}(v),
$$

that is integral over $\mathbb{O}$ (its coefficients satisfy $a, c \in \mathbb{Z}$ and $b \in \mathbb{O}$ ) and indefinite (its discriminant $\Delta(f)=\mathrm{n}(b)-a c$ is positive); see Section 4. We denote by $\mathrm{SL}_{2}(\mathrm{O})$ the group of invertible $2 \times 2$ matrices with coefficients in 0 ; see Section 3 . The group $\mathrm{SU}_{f}(\mathcal{O})$ of automorphs of $f$ consists of those elements $g \in \mathrm{SL}_{2}(\mathcal{O})$ for which $f \circ g=f$. Given an arithmetic group $\Gamma$, such as $\mathrm{SL}_{2}\left(O_{)}\right)$or $\mathrm{SU}_{f}(\mathcal{O})$, we will denote by $\operatorname{Covol}(\Gamma)$ the volume of the quotient by $\Gamma$ of its associated symmetric space (assumed to be of noncompact type and normalized to have -1 as the maximum of its sectional curvature).

For every $s>0$, we consider the integer

$$
\psi_{f, \mathfrak{m}}(s)=\operatorname{Card}_{\mathrm{SU}_{f}(\mathcal{O})} \backslash\left\{(u, v) \in \mathfrak{m} \times \mathfrak{m}: \mathrm{n}(\mathfrak{m})^{-1}|f(u, v)| \leq s, \mathcal{O} u+\mathscr{O} v=\mathfrak{m}\right\},
$$

which is the number of nonequivalent $\mathfrak{m}$-primitive representations by $f$ of rational integers with absolute value at most $s$. The finiteness of $\psi_{f, \mathfrak{m}}(s)$ follows from general results on orbits of algebraic groups defined over number fields [Borel and Harish-Chandra 1962, Lemma 5.3].

Theorem 1. As $s$ tends to $+\infty$, we have the equivalence, with $p$ ranging over positive rational primes,

$$
\psi_{f, \mathfrak{m}}(s) \sim \frac{45 D_{A} \operatorname{Covol}\left(\operatorname{SU}_{f}(\mathcal{O})\right)}{2 \pi^{2} \zeta(3) \Delta(f)^{2} \prod_{p \mid D_{A}}\left(p^{3}-1\right)} s^{4}
$$


This result follows from the more general Theorem 13, which allows us in particular to count representations satisfying given congruence properties (see the end of Section 6).

Here is an example of our applications, concerning the asymptotic of the very useful real scalar product $(u, v) \mapsto \operatorname{tr}(\bar{u} v)$ on $\mathbb{H}$. See Section 6 for the proof and for further applications. Let

$$
\mathrm{Sp}_{1}(\mathcal{O})=\left\{g \in \mathrm{SL}_{2}(\mathcal{O}):{ }^{t} \bar{g}\left(\begin{array}{ll}
0 & 1 \\
1 & 0
\end{array}\right) g=\left(\begin{array}{ll}
0 & 1 \\
1 & 0
\end{array}\right)\right\} .
$$

Corollary 2. As s tends to $+\infty$, we have the equivalence

Card $\operatorname{Sp}_{1}(\mathcal{O}) \backslash\{(u, v) \in \mathcal{O} \times \mathbb{O}:|\operatorname{tr}(\bar{u} v)| \leq s, \mathcal{O} u+\mathcal{O} v=\mathcal{O}\} \sim \frac{D_{A}}{48 \zeta(3)} \prod_{p \mid D_{A}} \frac{p^{2}+1}{p^{2}+p+1} s^{4}$.

To prove Theorem 1, applying a counting result of [Parkkonen and Paulin 2012] following from dynamical properties of the geodesic flow of real hyperbolic manifolds, we first prove that

$$
\psi_{f, \mathfrak{m}}(s) \sim \frac{D_{A} \prod_{p \mid D_{A}}(p-1) \operatorname{Covol}\left(\mathrm{SU}_{f}(\mathcal{O})\right)}{512 \pi^{2} \Delta(f)^{2} \operatorname{Covol}\left(\mathrm{SL}_{2}(\mathcal{O})\right)} s^{4} .
$$

The covolumes of the arithmetic groups $\mathrm{SL}_{2}(\mathrm{O})$ and $\mathrm{SU}_{f}(\mathrm{O})$ may be computed using the very general formula of [Prasad 1989]; see [Emery 2009] for an excellent exposition. Following the approach of [Rankin 1939a; 1939b; Selberg 1940], see also [Langlands 1966; Sarnak 1983] and others, we compute $\operatorname{Covol}\left(\mathrm{SL}_{2}(\mathbf{O})\right)$ in the main body of this paper (see Section 5) using Eisenstein series, whose analytic properties in the quaternion setting have been studied in [Krafft and Osenberg 1990]. We initially proved the case $h_{A}=1$ of the following result, V. Emery proved the general case using Prasad's formula (see the appendix), and we afterwards managed to push the Eisenstein series approach to get the general result. The two proofs are completely different.

Theorem 3 (Emery; see the appendix). We have

$$
\operatorname{Covol}\left(\mathrm{SL}_{2}(\mathrm{O})\right)=\frac{\zeta(3) \prod_{p \mid D_{A}}\left(p^{3}-1\right)(p-1)}{11520} .
$$

In the final section, we give a geometric reduction theory of binary Hamiltonian forms using real hyperbolic geometry. The case of binary quadratic forms is well known, from either the arithmetic, geometric or algorithmic viewpoint; see for instance [Cassels 1978; Zagier 1981; Buchmann and Vollmer 2007]. We refer for instance to [Elstrodt et al. 1998] for the reduction theory of binary Hermitian forms. The case of binary Hamiltonian forms has been developed less; see for instance [Pronin 1967; Hashimoto and Ibukiyama 1980; 1981; 1983] for results 
in the positive definite case. We construct a natural map $\Xi$ from the set $2(\mathbb{O}, \Delta)$ of binary Hamiltonian forms that are integral over 0 and have a fixed discriminant $\Delta \in \mathbb{Z}-\{0\}$ to the set of points or totally geodesic hyperplanes of the 5-dimensional real hyperbolic space $\mathbb{H}_{\mathbb{R}}^{5}$. For $\mathscr{F}_{\mathbb{O}}$ a Ford fundamental domain for the action of $\mathrm{SL}_{2}(O)$ on $\mathbb{T}_{\mathbb{R}}^{5}$, we say that $f \in 2(O, \Delta)$ is reduced if $\Xi(f)$ meets $\mathscr{F}_{\mathbb{O}}$. The finiteness of the number of orbits of $\mathrm{SL}_{2}(\mathrm{O})$ on $2(0, \Delta)$, which can be deduced from general results of Borel and Harish-Chandra, then follows in an explicit way from the equivariance property of $\Xi$ and the following result proved in Section 7 .

Theorem 4. There are only finitely many reduced integral binary Hamiltonian forms with a fixed nonzero discriminant.

Answering the remark on page 257 of [Cassels 1978] that explicit sets of inequalities implying the reduction property were essentially only known for quadratic forms in dimension $n \leq 7$, we give an explicit such set in dimension 8 at the end of Section 7.

The knowledgeable reader may skip the background Sections 2 (except the new Lemma 6), 3 and 4 on respectively definite quaternion algebras over $\mathbb{Q}$, quaternionic homographies and real hyperbolic geometry in dimension 5, and binary Hamiltonian forms, though many references are made to them in the subsequent sections.

\section{Background on definite quaternion algebras over $\mathbb{Q}$}

A quaternion algebra over a field $F$ is a four-dimensional central simple algebra over $F$. We refer for instance to [Vignéras 1980] for generalities on quaternion algebras.

A real quaternion algebra is isomorphic either to $\mu_{2}(\mathbb{R})$ or to Hamilton's quaternion algebra $\mathbb{W}$ over $\mathbb{R}$, with basis elements $1, i, j, k$ as a $\mathbb{R}$-vector space, with unit element 1 , satisfying $i^{2}=j^{2}=-1$ and $i j=-j i=k$. We define the conjugate of $x=$ $x_{0}+x_{1} i+x_{2} j+x_{3} k$ in $\mathbb{H}$ by $\bar{x}=x_{0}-x_{1} i-x_{2} j-x_{3} k$, its reduced trace by $\operatorname{tr}(x)=x+\bar{x}$, and its reduced norm by $\mathrm{n}(x)=x \bar{x}=\bar{x} x$. Note that $\mathrm{n}(x y)=\mathrm{n}(x) \mathrm{n}(y)$, and $\mathrm{n}(x) \geq 0$ with equality if and only if $x=0$; hence $\mathbb{U}$ is a division algebra. Furthermore, $\operatorname{tr}(\bar{x})=\operatorname{tr}(x)$ and $\operatorname{tr}(x y)=\operatorname{tr}(y x)$. For every matrix $X=\left(x_{i, j}\right)_{1 \leq i \leq p, 1 \leq j \leq q} \in M_{p, q}(\mathbb{W})$, we denote by $X^{*}=\left(\overline{x_{j, i}}\right)_{1 \leq i \leq q, 1 \leq j \leq p} \in \mathcal{M}_{q, p}(\mathbb{U})$ its adjoint matrix, which satisfies $(X Y)^{*}=Y^{*} X^{*}$. The matrix $X$ is Hermitian if $X=X^{*}$.

Let $A$ be a quaternion algebra over $\mathbb{Q}$. We say that $A$ is definite (or ramified over $\mathbb{R}$ ) if the real quaternion algebra $A \otimes_{\mathbb{Q}} \mathbb{R}$ is isomorphic to $\mathbb{H}$. In this paper, whenever we consider a definite quaternion algebra $A$ over $\mathbb{Q}$, we will fix an identification between $A \otimes_{\mathbb{Q}} \mathbb{R}$ and $\mathbb{H}$, so that $A$ is a $\mathbb{Q}$-subalgebra of $\mathbb{W}$.

The reduced discriminant $D_{A}$ of $A$ is the product of the primes $p \in \mathbb{N}$ such that the quaternion algebra $A \otimes_{\mathbb{Q}} \mathbb{Q}_{p}$ over $\mathbb{Q}_{p}$ is a division algebra, with $\left[\mathbb{W}^{\times}, \mathbb{H}^{\times}\right]=\mathrm{n}^{-1}(1)$. Two definite quaternion algebras over $\mathbb{Q}$ are isomorphic if and only if they have the 
same reduced discriminant, which can be any product of an odd number of primes; see [Vignéras 1980, page 74].

A $\mathbb{Z}$-lattice $I$ in $A$ is a finitely generated $\mathbb{Z}$-module generating $A$ as a $\mathbb{Q}$-vector space. The intersection of finitely many $\mathbb{Z}$-lattices of $A$ is again a $\mathbb{Z}$-lattice. An order in a quaternion algebra $A$ over $\mathbb{Q}$ is a unitary subring $O$ of $A$ which is a $\mathbb{Z}$-lattice. In particular, $A=\mathbb{Q} O$. Each order of $A$ is contained in a maximal order. The type number $t_{A} \geq 1$ of $A$ is the number of conjugacy (or equivalently isomorphism) classes of maximal orders in $A$ (see for instance [Vignéras 1980, page 152] for a formula). For instance, $t_{A}=1$ if $D_{A}=2,3,5,7,13$ and $t_{A}=2$ if $D_{A}=11,17$. If 0 is a maximal order in $A$, then the ring $O$ has 2,4 or 6 invertible elements except that $\left|0^{\times}\right|=24$ when $D_{A}=2$, and $\left|0^{\times}\right|=12$ when $D_{A}=3$. When $D_{A}=2,3,5,7,13$, then (see [Eichler 1938, page 103])

$$
\left|0^{\times}\right|=\frac{24}{D_{A}-1} \text {. }
$$

Example 5 (See [Vignéras 1980, page 98]).

(1) The $\mathbb{Q}$-vector space $A=\mathbb{Q}+\mathbb{Q} i+\mathbb{Q} j+\mathbb{Q} k$ generated by $1, i, j, k$ in $\mathbb{H}$ is Hamilton's quaternion algebra over $\mathbb{Q}$. It is the unique definite quaternion algebra over $\mathbb{Q}$ (up to isomorphism) with discriminant $D_{A}=2$. The Hurwitz order $\mathbb{O}=\mathbb{Z}+\mathbb{Z} i+\mathbb{Z} j+\mathbb{Z}(1+i+j+k) / 2$ is maximal, and it is unique up to conjugacy.

(2) Similarly, $A=\mathbb{Q}+\mathbb{Q} i+\mathbb{Q} \sqrt{p} j+\mathbb{Q} \sqrt{p} k$ is the unique (up to isomorphism) definite quaternion algebra over $\mathbb{Q}$ with discriminant $D_{A}=p$ for $p=3,7$, and $0=\mathbb{Z}+\mathbb{Z} i+\mathbb{Z}(i+\sqrt{p} j) / 2+\mathbb{Z}(1+\sqrt{p} k) / 2$ is its unique (up to conjugacy) maximal order.

(3) Similarly, $A=\mathbb{Q}+\mathbb{Q} \sqrt{2} i+\mathbb{Q} \sqrt{p} j+\mathbb{Q} \sqrt{2 p} k$ is the unique (up to isomorphism) definite quaternion algebra over $\mathbb{Q}$ with discriminant $D_{A}=p$ for $p=5,13$, and

$$
0=\mathbb{Z}+\mathbb{Z} \frac{1+\sqrt{2} i+\sqrt{p} j}{2}+\mathbb{Z} \frac{\sqrt{p} j}{2}+\mathbb{Z} \frac{2+\sqrt{2} i+\sqrt{2 p} k}{2}
$$

is its unique (up to conjugacy) maximal order.

Let $O$ be an order in $A$. The reduced norm $\mathrm{n}$ and the reduced trace tr take integral values on 0 . The invertible elements of $O$ are its elements of reduced norm 1 . Since $\bar{x}=\operatorname{tr}(x)-x$, any order is invariant under conjugation.

The left order $\mathrm{O}_{\ell}(I)$ of a $\mathbb{Z}$-lattice $I$ is $\{x \in A: x I \subset I\}$; its right order $\mathrm{O}_{r}(I)$ is $\{x \in A: I x \subset I\}$. A left fractional ideal of $O$ is a $\mathbb{Z}$-lattice of $A$ whose left order is $\mathbb{O}$. A left ideal of $\mathbb{O}$ is a left fractional ideal of $O$ contained in $O$. Right (fractional) ideals are defined analogously. The inverse of a right fractional ideal $\mathfrak{m}$ of $\mathbb{O}$ is 
$\mathfrak{m}^{-1}=\{x \in A: \mathfrak{m} x \mathfrak{m} \subset \mathfrak{m}\}$. It is easy to check that for every $u, v \in \mathcal{O}$, if $u v \neq 0$, then

$$
(u \mathscr{O}+v \mathscr{O})^{-1}=\mathscr{O} u^{-1} \cap \mathcal{O} v^{-1} .
$$

If $\mathcal{O}$ is maximal, then $\mathfrak{m}^{-1}$ is a left fractional ideal of $\mathcal{O}$ and

$$
O_{r}\left(\mathfrak{m}^{-1}\right)=O_{\ell}(\mathfrak{m})
$$

This formula follows from [Vignéras 1980, Lemma 4.3(3), page 21], which says that $O_{r}\left(\mathfrak{m}^{-1}\right)$ contains $\mathrm{O}_{\ell}(\mathfrak{m})$, since the maximality of 0 implies the maximality of $\mathrm{O}_{\ell}(\mathfrak{m})$, by [ibid., Exercice 4.1, page 28].

Two left fractional ideals $\mathfrak{m}$ and $\mathfrak{m}^{\prime}$ of $\mathbb{O}$ are isomorphic as left $\mathbb{O}$-modules if and only if $\mathfrak{m}^{\prime}=\mathfrak{m} c$ for some $c \in A^{\times}$. A (left) ideal class of $\mathcal{O}$ is an equivalence class of left fractional ideals of $O$ for this equivalence relation. We will denote by ${ }_{0} \mathscr{I}$ the set of ideal classes of 0 , and by $[\mathfrak{m}]$ the ideal class of a left fractional ideal $\mathfrak{m}$ of 0 . The class number $h_{A}$ of $A$ is the number of ideal classes of a maximal order 0 of $A$. It is finite and independent of the maximal order $\mathcal{O}$; see for instance [ibid., pages 87-88]. See for instance [ibid., pages 152-155] for a formula for $h_{A}$, and for the fact that $h_{A}=1$ if and only if $D_{A}=2,3,5,7,13$. In particular $D_{A}$ is prime if $h_{A}=1$.

The norm $\mathrm{n}(\mathfrak{m})$ of a left (or right) ideal $\mathfrak{m}$ of $\mathbb{O}$ is the greatest common divisor of the norms of the nonzero elements of $\mathfrak{m}$. In particular, $\mathrm{n}(\mathbb{O})=1$. The norm of a left (or right) fractional ideal $\mathfrak{m}$ of $O$ is $n(c \mathfrak{m}) / \mathrm{n}(c)$ for any $c \in \mathbb{N}-\{0\}$ such that $c \mathfrak{m} \subset \mathbb{O}$.

Note that a $\mathbb{Z}$-lattice $\Lambda$ in $A$ is a $\mathbb{Z}$-lattice in the Euclidean vector space $\mathbb{M}$ (with orthonormal basis $(1, i, j, k)$ ), and the volume $\operatorname{Vol}(\Lambda \backslash \mathbb{M})$ is finite. If $\mathbb{O}$ is maximal, we have (see for instance [Krafft and Osenberg 1990, Lemma 5.5])

$$
\operatorname{Vol}(\mathbb{O} \backslash \mathbb{M})=\frac{D_{A}}{4}
$$

The classical zeta function of $A$ is

$$
\zeta_{A}(s)=\sum_{\mathfrak{a}} \frac{1}{\mathrm{n}(\mathfrak{a})^{2 s}},
$$

where the sum is over all left ideals $\mathfrak{a}$ in a maximal order $O$ of $A$. It is independent of the choice of $\mathbb{O}$, it is holomorphic on $\{s \in \mathbb{C}: \operatorname{Re} s>1\}$ and it satisfies by a theorem of Hey, with $\zeta$ the usual Riemann zeta function,

$$
\zeta_{A}(s)=\zeta(2 s) \zeta(2 s-1) \prod_{p \mid D_{A}}\left(1-p^{1-2 s}\right),
$$


where as usual the index $p$ is prime; see [Schoeneberg 1939, page 88; Vignéras 1980, page 64]. Let $\mathfrak{m}$ be a left fractional ideal of a maximal order $\mathcal{O}$ in $A$. Define

$$
\zeta(\mathfrak{m}, s)=\mathrm{n}(\mathfrak{m})^{2 s} \sum_{x \in \mathfrak{m}-\{0\}} \frac{1}{\mathrm{n}(x)^{2 s}},
$$

which is also holomorphic on $\operatorname{Re} s>1$ (and depends only on the ideal class of $\mathfrak{m}$ ), and

$$
\zeta_{[\mathfrak{m}]}(s)=\sum \frac{1}{\mathrm{n}(\mathfrak{a})^{2 s}}
$$

where the sum is over all left ideals $\mathfrak{a}$ in 0 whose ideal class is $[\mathfrak{m}]$. The relations we will use in Section 5 between these zeta functions are the following ones, where $\operatorname{Re} s>1$. The first one is obvious; see for instance respectively [Deuring 1968, page 134] and [Krafft and Osenberg 1990, page 436] for the other two:

$$
\begin{aligned}
& \zeta_{A}(s)=\sum_{[\mathfrak{a}] \in_{\mathscr{O}} \mathscr{I}} \zeta_{[\mathfrak{a}]}(s), \\
& \sum_{[\mathfrak{a}] \in_{\mathfrak{O}} \mathscr{I}} \frac{1}{\left|\mathcal{O}_{r}(\mathfrak{a})^{\times}\right|}=\frac{1}{24} \prod_{p \mid D_{A}}(p-1), \\
& \zeta(\mathfrak{m}, s)=\left|\mathscr{O}_{r}(\mathfrak{m})^{\times}\right| \zeta_{[\mathfrak{m}]}(s) .
\end{aligned}
$$

Note that when the class number $h_{A}$ of $A$ is 1 , the formula (9) becomes

$$
\zeta(O, s)=\left|O^{\times}\right| \zeta_{A}(s)
$$

We end this section with the following lemma, which will be used in the proof of Theorem 13.

Lemma 6. Let $\mathbb{O}$ be a maximal order in a definite quaternion algebra $A$ over $\mathbb{Q}$, let

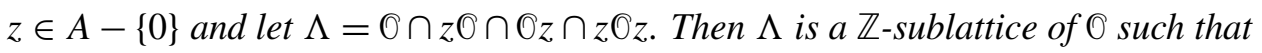

$$
[0: \Lambda] \mathrm{n}\left(0 z^{-1}+0\right)^{4}=1 .
$$

Proof. This is a "prime by prime" type of proof, suggested by G. Chenevier. As an intersection of four $\mathbb{Z}$-lattices, $\Lambda$ is a $\mathbb{Z}$-lattice, contained in $\mathbb{O}$. For every (positive rational) prime $p$, let $v_{p}$ be the $p$-adic valuation on $\mathbb{Q}_{p}$; let us consider the quaternion algebra $A_{p}=A \otimes_{\mathbb{Q}} \mathbb{Q}_{p}$ over $\mathbb{Q}_{p}$, whose reduced norm is denoted by $\mathrm{n}_{p}: A_{p} \rightarrow \mathbb{Q}_{p}$; and for every $\mathbb{Z}$-lattice $L$ of $A$, let $L_{p}=L \otimes_{\mathbb{Z}} \mathbb{Z}_{p}$. We embed $A$ in $A_{p}$ as usual by $x \mapsto x \otimes 1$. We then have the following properties (see for instance [Vignéras 1980, page 83-84]): $L_{p}$ is a $\mathbb{Z}_{p}$-lattice of $A_{p}$; the map $L \mapsto L_{p}$ commutes with the inclusion, the sum and the intersection; if $L$ and $L^{\prime}$ are $\mathbb{Z}$-lattices with $L \subset L^{\prime}$, then

$$
\left[L^{\prime}: L\right]=\prod_{p}\left[L_{p}^{\prime}: L_{p}\right]
$$


if $L$ is a left fractional ideal of $O_{\text {, then }} L_{p}$ is a left fractional ideal of $O_{p}$, and

$$
\mathrm{n}(L)=\prod_{p} p^{v_{p}\left(\mathrm{n}_{p}\left(L_{p}\right)\right)} .
$$

Hence in order to prove Lemma 6 , we only have to prove that for every prime $p$, if $z \in A_{p}^{\times}$and $\Lambda_{p}=\mathfrak{O}_{p} \cap z \mathscr{O}_{p} \cap \mathfrak{O}_{p} z \cap z \mathbb{O}_{p} z$, we have

$$
\left[\mathcal{O}_{p}: \Lambda_{p}\right]=p^{-4 v_{p}\left(\mathrm{n}_{p}\left(\mathscr{O}_{p} z^{-1}+\mathscr{O}_{p}\right)\right)} .
$$

We distinguish two cases.

First assume that $p$ does not divide $D_{A}$. Then we may assume that $A_{p}=M_{2}\left(\mathbb{Q}_{p}\right)$ and $\mathbb{O}_{p}=M_{2}\left(\mathbb{Z}_{p}\right)$ (by the uniqueness up to conjugacy of maximal orders). By Cartan's decomposition of $\mathrm{GL}_{2}\left(\mathbb{Q}_{p}\right)$ (see for instance [Bruhat and Tits 1972], or consider the action of $\mathrm{GL}_{2}\left(\mathbb{Q}_{p}\right)$ on its Bruhat-Tits tree as in [Serre 1977]), the element $z \in \mathrm{GL}_{2}\left(\mathbb{Q}_{p}\right)$ may be written

$$
z=P\left(\begin{array}{cc}
p^{a} & 0 \\
0 & p^{b}
\end{array}\right) Q
$$

with $P, Q$ in the (good) maximal compact subgroup $\mathrm{GL}_{2}\left(\mathbb{Z}_{p}\right)$ and $a, b$ in $\mathbb{Z}$. Since $\mathrm{GL}_{2}\left(\mathbb{Z}_{p}\right)$ preserves $\mathrm{O}_{p}=M_{2}\left(\mathbb{Z}_{p}\right)$ by left and right multiplication, preserves the indices of $\mathbb{Z}$-lattices, and contains only elements of reduced norm (that is, of determinant) having valuation 0 , we may assume that $P=Q=$ id. We hence have, by an easy matrix computation,

$$
\begin{aligned}
\Lambda_{p} & =\left(\begin{array}{cc}
\mathbb{Z}_{p} \cap p^{a} \mathbb{Z}_{p} \cap p^{2 a} \mathbb{Z}_{p} & \mathbb{Z}_{p} \cap p^{a} \mathbb{Z}_{p} \cap p^{b} \mathbb{Z}_{p} \cap p^{a+b} \mathbb{Z}_{p} \\
\mathbb{Z}_{p} \cap p^{a} \mathbb{Z}_{p} \cap p^{b} \mathbb{Z}_{p} \cap p^{a+b} \mathbb{Z}_{p} & \mathbb{Z}_{p} \cap p^{b} \mathbb{Z}_{p} \cap p^{2 b} \mathbb{Z}_{p}
\end{array}\right) \\
& =\left(\begin{array}{cc}
p^{2 \max \{a, 0\}} \mathbb{Z}_{p} & p^{\max \{a, 0\}+\max \{b, 0\}} \mathbb{Z}_{p} \\
p^{\max \{a, 0\}+\max \{b, 0\}} \mathbb{Z}_{p} & p^{2 \max \{b, 0\}} \mathbb{Z}_{p}
\end{array}\right) .
\end{aligned}
$$

Similarly, we have

$$
\mathcal{O}_{p} z^{-1}+\mathcal{O}_{p}=\left(\begin{array}{ll}
p^{-a} \mathbb{Z}_{p}+\mathbb{Z}_{p} & p^{-b} \mathbb{Z}_{p}+\mathbb{Z}_{p} \\
p^{-a} \mathbb{Z}_{p}+\mathbb{Z}_{p} & p^{-b} \mathbb{Z}_{p}+\mathbb{Z}_{p}
\end{array}\right)=M_{2}\left(\mathbb{Z}_{p}\right)\left(\begin{array}{cc}
p^{\min \{-a, 0\}} & 0 \\
0 & p^{\min \{-b, 0\}}
\end{array}\right) .
$$

Therefore, since $\mathrm{n}_{p}\left(M_{2}\left(\mathbb{Z}_{p}\right)\right)=1$ and $\mathrm{n}_{p}=\operatorname{det}$ on $A_{p}=M_{2}\left(\mathbb{Q}_{p}\right)$,

$$
\begin{aligned}
& {\left[\mathscr{O}_{p}: \Lambda_{p}\right]} \\
& \quad=\left|\mathbb{Z}_{p} /\left(p^{2 \max \{a, 0\}} \mathbb{Z}_{p}\right)\right|\left|\mathbb{Z}_{p} /\left(p^{\max \{a, 0\}+\max \{b, 0\}} \mathbb{Z}_{p}\right)\right|^{2}\left|\mathbb{Z}_{p} /\left(p^{2 \max \{b, 0\}} \mathbb{Z}_{p}\right)\right| \\
& \quad=p^{4(\max \{a, 0\}+\max \{b, 0\})}=p^{-4(\min \{-a, 0\}+\min \{-b, 0\})}=p^{-4 v_{p}\left(\mathrm{n}_{p}\left(\mathscr{C}_{p} z^{-1}+\mathscr{O}_{p}\right)\right)},
\end{aligned}
$$

as wanted.

Now assume that $p$ divides $D_{A}$, so that $A_{p}$ is a division algebra. Let $v=v_{p} \circ \mathrm{n}_{p}$, which, by for instance [Vignéras 1980, page 34], is a discrete valuation on $A_{p}$, 
whose valuation ring is $O_{p}$. The left ideals of $O_{p}$ are two-sided ideals. Let $\pi$ be a uniformizer of $\sigma_{p}$. Note that the residual field $\sigma_{p} / \pi \mathcal{O}_{p}$ has order $p^{2}$, and that $\mathrm{n}_{p}\left(\mathscr{O}_{p}\right)=1$ and $\mathrm{n}_{p}(\pi)=p$. We have

$$
\Lambda_{p}=\mathfrak{O}_{p} \pi^{2 \max \{v(z), 0\}} \quad \text { and } \quad \mathfrak{O}_{p} z^{-1}+\mathscr{O}_{p}=\mathscr{O}_{p} \pi^{\min \left\{v\left(z^{-1}\right), 0\right\}} .
$$

Hence $\left[\mathcal{O}_{p}: \Lambda_{p}\right]=p^{4 \max \{v(z), 0\}}$ and $v_{p}\left(\mathrm{n}_{p}\left(\mathscr{O}_{p} z^{-1}+\mathscr{O}_{p}\right)\right)=-\max \{v(z), 0\}$, which is also as wanted.

\section{Background on Hamilton-Bianchi groups}

The Dieudonné determinant (see [Dieudonné 1943; Aslaksen 1996]) Det is the group morphism from the group $\mathrm{GL}_{2}(\mathbb{M})$ of invertible $2 \times 2$ matrices with coefficients in $\mathbb{H}$ to $\mathbb{R}_{+}^{*}$, defined by

$$
\begin{aligned}
\left(\operatorname{Det}\left(\begin{array}{ll}
a & b \\
c & d
\end{array}\right)\right)^{2} & =\mathrm{n}(a d)+\mathrm{n}(b c)-\operatorname{tr}(a \bar{c} d \bar{b}) \\
& = \begin{cases}\mathrm{n}\left(a d-a c a^{-1} b\right) & \text { if } a \neq 0, \\
\mathrm{n}\left(c b-c a c^{-1} d\right) & \text { if } c \neq 0 \\
\mathrm{n}\left(c b-d b^{-1} a b\right) & \text { if } b \neq 0\end{cases}
\end{aligned}
$$

It is invariant under the adjoint map $g \mapsto g^{*}$, by the properties of $\mathrm{n}$ and tr. We will denote by $\mathrm{SL}_{2}(\mathbb{H})$ the group of $2 \times 2$ matrices with coefficients in $\mathbb{H}$ with Dieudonné determinant 1 , which equals the group of elements of (reduced) norm 1 in the central simple algebra $M_{2}(\mathbb{M})$ over $\mathbb{R}$; see [Reiner 1975, Section 9a]. See [Kellerhals 2003] for more information on $\mathrm{SL}_{2}(\mathbb{M})$.

The group $\mathrm{SL}_{2}(\mathbb{H})$ acts linearly on the left on the right $\mathbb{M}$-module $\mathbb{H} \times \mathbb{H}$. Let $\mathbb{P}_{r}^{1}(\mathbb{H})=(\mathbb{H} \times \mathbb{W}-\{0\}) / \mathbb{W}^{\times}$be the right projective line of $\mathbb{H}$, identified as usual with the Alexandrov compactification $\mathbb{U} \cup\{\infty\}$ where $[1: 0]=\infty$ and $[x: y]=x y^{-1}$ if $y \neq 0$. The projective action of $\mathrm{SL}_{2}(\mathbb{M})$ on $\mathbb{P}_{r}^{1}(\mathbb{M})$, induced by its linear action on

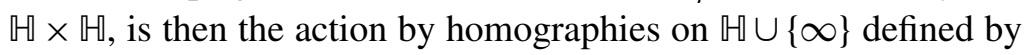

$$
\left(\begin{array}{ll}
a & b \\
c & d
\end{array}\right) \cdot z= \begin{cases}(a z+b)(c z+d)^{-1} & \text { if } z \neq \infty,-c^{-1} d \\
a c^{-1} & \text { if } z=\infty, c \neq 0 \\
\infty & \text { otherwise }\end{cases}
$$

This action induces a faithful left action of $\mathrm{PSL}_{2}(\mathbb{H})=\mathrm{SL}_{2}(\mathbb{U}) /\{ \pm$ id $\}$ on $\mathbb{U} \cup\{\infty\}$.

The group $\mathrm{PSL}_{2}(\mathbb{M})$ is very useful for studying 5-dimensional real hyperbolic geometry for the following reason. Let us endow $\mathbb{W}$ with its usual Euclidean metric $d s_{\llbracket}^{2}$ (invariant under translations, with $(1, i, j, k)$ orthonormal). We will denote by $x=(z, r)$ a generic point in $\mathbb{H} \times] 0,+\infty[$, and by $r: x \mapsto r(x)$ the second projection in this product. For the real hyperbolic space $\mathbb{T}_{\mathbb{R}}^{5}$ of dimension 5 , 
we will use the upper halfspace model $\mathbb{\|} \times] 0,+\infty[$ with Riemannian metric $d s^{2}(x)=\left(d s_{\llbracket \rrbracket}^{2}(z)+d r^{2}\right) / r^{2}$ at the point $x=(z, r)$, whose volume form is

$$
d \operatorname{vol}_{\mathbb{Q} \mathbb{R}_{\mathbb{R}}^{5}}(x)=\frac{d \operatorname{vol}_{\mathbb{W}}(z) d r}{r^{5}} .
$$

The space at infinity $\partial_{\infty} \mathbb{H}_{\mathbb{R}}^{5}$ is hence $\mathbb{U} \cup\{\infty\}$.

By the Poincaré extension procedure (see for instance [Parkkonen and Paulin 2010, Lemma 6.6]), the action of $\mathrm{SL}_{2}(\mathbb{H})$ by homographies on $\partial_{\infty} \mathbb{T}_{\mathbb{R}}^{5}$ extends to a left action on $\mathbb{U}_{\mathbb{R}}^{5}$ by

$$
\left(\begin{array}{ll}
a & b \\
c & d
\end{array}\right) \cdot(z, r)=\left(\frac{(a z+b) \overline{(c z+d)}+a \bar{c} r^{2}}{\mathrm{n}(c z+d)+r^{2} \mathrm{n}(c)}, \frac{r}{\mathrm{n}(c z+d)+r^{2} \mathrm{n}(c)}\right) .
$$

In this way, the group $\mathrm{PSL}_{2}(\mathbb{W})$ is identified with the group of orientation preserving isometries of $\mathbb{M}_{\mathbb{R}}^{5}$. Note that the isomorphism $\mathrm{PSL}_{2}(\mathbb{M}) \simeq \mathrm{SO}_{0}(1,5)$ is one of the isomorphisms between connected simple real Lie groups of small dimensions in E. Cartan's classification.

Given an order $\mathbb{O}$ in a definite quaternion algebra $A$ over $\mathbb{Q}$, define the HamiltonBianchi group as $\Gamma_{\mathscr{O}}=\mathrm{SL}_{2}(\mathbb{O})=\mathrm{SL}_{2}(\mathbb{Q}) \cap M_{2}(\mathbb{O})$. Note that since the norm $\mathrm{n}$ takes integral values on $\mathbb{O}$, and since the Dieudonné determinant is a group morphism, we have $\mathrm{GL}_{2}(\mathrm{O})=\mathrm{SL}_{2}(\mathrm{O})$. The Hamilton-Bianchi group $\Gamma_{\mathcal{O}}$ is a (nonuniform) arithmetic lattice in the connected real Lie group $\mathrm{SL}_{2}(\mathbb{H})$ (see for instance [Parkkonen and Paulin 2010, page 1104] for details). In particular, the quotient real hyperbolic orbifold $\Gamma_{\mathscr{O}} \backslash \mathbb{T}_{\mathbb{R}}^{5}$ has finite volume. The action by homographies of $\Gamma_{\mathscr{O}}$ preserves the right projective space $\mathbb{P}_{r}^{1}(\mathcal{O})=A \cup\{\infty\}$, which is the set of fixed points of the parabolic elements of $\Gamma_{\mathscr{O}}$ acting on $\mathbb{T}_{\mathbb{R}}^{5} \cup \partial_{\infty} \mathbb{R}_{\mathbb{R}}^{5}$.

Remark 7. For every $(u, v)$ in $\mathcal{O} \times \mathbb{O}-\{(0,0)\}$, consider the two left ideals of $\mathcal{O}$

$$
I_{u, v}=\mathscr{O} u+\mathscr{O} v \quad \text { and } \quad K_{u, v}= \begin{cases}\mathcal{O} u \cap \mathcal{O} v & \text { if } u v \neq 0 \\ \mathcal{O} & \text { otherwise }\end{cases}
$$

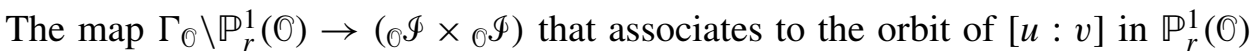
under $\Gamma_{\mathscr{O}}$ the couple of ideal classes $\left(\left[I_{u, v}\right],\left[K_{u, v}\right]\right)$ is a bijection. To see this, let $\ell_{u, v}: 0 \times \mathbb{O} \rightarrow \mathbb{O}$ be the morphism of left $\mathbf{O}$-modules defined by $\left(o_{1}, o_{2}\right) \mapsto$ $o_{1} u+o_{2} v$. The map $w \mapsto\left(w u^{-1},-w v^{-1}\right)$ is an isomorphism of left 0 -modules from $0 u \cap \mathcal{O} v$ to the kernel of $\ell_{u, v}$ if $u v \neq 0$. The result then follows for instance from [Krafft and Osenberg 1990, Satz 2.1, 2.2], which says that the map $[u: v] \mapsto$ $\left(\left[\operatorname{im} \ell_{u, v}\right],\left[\operatorname{ker} \ell_{u, v}\right]\right)$ induces a bijection from $\Gamma_{\mathscr{O}} \backslash \mathbb{P}_{r}^{1}(\mathcal{O})$ into $\mathscr{O}^{\mathscr{T}} \times{ }_{\mathbb{O}} \mathscr{I}$.

In particular, the number of cusps of $\Gamma_{\mathscr{O}}$ (or the number of ends of $\Gamma_{\mathscr{O}} \backslash \mathbb{Q} \mathbb{R}_{\mathbb{R}}^{5}$ ) is the square of the class number $h_{A}$ of $A$. 


\section{Background on binary Hamiltonian forms}

With $V$ the right $\mathbb{M}$-module $\mathbb{U} \times \mathbb{H}$, a binary Hamiltonian form $f: V \rightarrow \mathbb{R}$ is a map $X \mapsto \phi(X, X)$ where $\phi: V \times V \rightarrow \mathbb{H}$ is a Hermitian form on $V$ with the conjugation as the anti-involution of the ring $\mathbb{H}$. That is, $\phi(X \lambda, Y)=\bar{\lambda} \phi(X, Y)$, $\phi\left(X+X^{\prime}, Y\right)=\phi(X, Y)+\phi\left(X^{\prime}, Y\right), \phi(Y, X)=\overline{\phi(X, Y)}$ for $X, X^{\prime}, Y \in V$ and $\lambda \in \mathbb{H}$. Our convention of sesquilinearity on the left is the opposite of Bourbaki's unfortunate one in [Bourbaki 1959]. Equivalently, a binary Hamiltonian form $f$ is a map $\mathbb{H} \times \mathbb{H} \rightarrow \mathbb{R}$ with

$$
f(u, v)=a \mathrm{n}(u)+\operatorname{tr}(\bar{u} b v)+c \mathrm{n}(v),
$$

whose coefficients $a=a(f)$ and $c=c(f)$ are real, and $b=b(f)$ lies in $\mathbb{M}$. Note that $f((u, v) \lambda)=\mathrm{n}(\lambda) f(u, v)$. The matrix $M(f)$ of $f$ is the Hermitian matrix

$$
\left(\begin{array}{ll}
a & b \\
\bar{b} & c
\end{array}\right)
$$

so that

$$
f(u, v)=\left(\begin{array}{l}
u \\
v
\end{array}\right)^{*}\left(\begin{array}{ll}
a & b \\
\bar{b} & c
\end{array}\right)\left(\begin{array}{l}
u \\
v
\end{array}\right) .
$$

The discriminant of $f$ is

$$
\Delta=\Delta(f)=\mathrm{n}(b)-a c .
$$

Note that the sign convention of the discriminant varies in the references. An easy computation shows that the Dieudonné determinant of $M(f)$ is equal to $|\Delta|$. If $a \neq 0$, then

$$
f(u, v)=a\left(\mathrm{n}\left(u+\frac{b v}{a}\right)-\frac{\Delta}{a^{2}} \mathrm{n}(v)\right) .
$$

Hence the form $f$ is indefinite (that is, $f$ takes both positive and negative values) if and only if $\Delta$ is positive, and $\Delta$ is then equal to the Dieudonné determinant of $M(f)$. By (15), the form $f$ is positive definite (that is, $f(x) \geq 0$ with equality if and only if $x=0$ ) if and only if $a>0$ and $\Delta<0$.

The linear action on the left on $\mathbb{W} \times \mathbb{W}$ of the group $\mathrm{SL}_{2}(\mathbb{W})$ induces an action on the right on the set of binary Hermitian forms $f$ by precomposition, that is, by $f \mapsto f \circ g$ for every $g \in \mathrm{SL}_{2}(\mathbb{H})$. The matrix of $f \circ g$ is $M(f \circ g)=g^{*} M(f) g$. Since the Dieudonné determinant is a group morphism, invariant under the adjoint map (and since $f \circ g$ is indefinite if and only if $f$ is), we have, for every $g \in \mathrm{SL}_{2}(\mathbb{W})$,

$$
\Delta(f \circ g)=\Delta(f) .
$$

Given an order $\mathbb{O}$ in a definite quaternion algebra over $\mathbb{Q}$, a binary Hamiltonian form $f$ is integral over 0 if its coefficients belong to $\mathbb{O}$. Note that such a form $f$ 
takes integral values on $0 \times 0$. The lattice $\Gamma_{\mathbb{O}}=\mathrm{SL}_{2}(\mathrm{O})$ of $\mathrm{SL}_{2}(\mathbb{U})$ preserves the set of indefinite binary Hamiltonian forms $f$ that are integral over 0 . The stabilizer in $\Gamma_{0}$ of such a form $f$ is its group of automorphs

$$
\mathrm{SU}_{f}(\mathcal{O})=\left\{g \in \Gamma_{\mathscr{O}}: f \circ g=f\right\} .
$$

For every indefinite binary Hamiltonian form $f$, with $a=a(f), b=b(f)$ and $\Delta=\Delta(f)$, let

$$
\begin{aligned}
\mathscr{C}_{\infty}(f) & =\left\{[u: v] \in \mathbb{P}_{r}^{1}(\mathbb{M}): f(u, v)=0\right\}, \\
\mathscr{C}(f) & =\{(z, r) \in \mathbb{M} \times] 0,+\infty\left[: f(z, 1)+a r^{2}=0\right\} .
\end{aligned}
$$

In $\mathbb{P}_{r}^{1}(\mathbb{M})=\mathbb{U} \cup\{\infty\}$, the set $\mathscr{C}_{\infty}(f)$ is the 3 -sphere of center $-b / a$ and radius $\sqrt{\Delta} /|a|$ if $a \neq 0$, and it is the union of $\{\infty\}$ with the real hyperplane

$$
\{z \in \mathbb{H}: \operatorname{tr}(\bar{z} b)+c=0\}
$$

of $\mathbb{H}$ otherwise. The map $f \mapsto \mathscr{C}_{\infty}(f)$ induces a bijection between the set of indefinite binary Hamiltonian forms up to multiplication by a nonzero real factor and the set of 3-spheres and real hyperplanes in $\mathbb{U} \cup\{\infty\}$. The action of $\mathrm{SL}_{2}(\mathbb{M})$

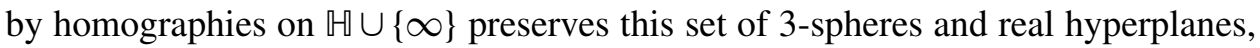
and the map $f \mapsto \mathscr{C}_{\infty}(f)$ is (anti)equivariant for the two actions of $\mathrm{SL}_{2}(\mathbb{I})$, in the sense that, for every $g \in \mathrm{SL}_{2}(\mathbb{H})$,

$$
\mathscr{C}_{\infty}(f \circ g)=g^{-1} \mathscr{C}_{\infty}(f) .
$$

Given a finite index subgroup $G$ of $\mathrm{SL}_{2}(\mathrm{O})$, an integral binary Hamiltonian form $f$ is called $G$-reciprocal if there exists an element $g$ in $G$ such that $f \circ g=-f$. We define $R_{G}(f)=2$ if $f$ is $G$-reciprocal, and $R_{G}(f)=1$ otherwise. The values of $f$ are positive on one of the two components of $\mathbb{P}_{r}^{1}(\mathbb{M})-\mathscr{C}_{\infty}(f)$ and negative on the other. As the signs are switched by precomposition by an element $g$ as above, the $G$-reciprocity of the form $f$ is equivalent to saying that there exists an element of $G$ preserving $\mathscr{C}_{\infty}(f)$ and exchanging the two complementary components of $\mathscr{C}_{\infty}(f)$.

\section{Using Eisenstein series to compute hyperbolic volumes}

Let $O$ be a maximal order in a definite quaternion algebra $A$ over $\mathbb{Q}$.

In this section, we compute $\operatorname{Vol}\left(\mathrm{PSL}_{2}(\mathcal{O}) \backslash \mathbb{U}_{\mathbb{R}}^{5}\right)$ using a method which goes back, in dimension 2, to Rankin and Selberg's method [Rankin 1939b; Selberg 1940] of integrating Eisenstein series on fundamental domains and "unfolding", generalized by [Langlands 1966] to the lattice of $\mathbb{Z}$-points of any connected split semisimple algebraic group over $\mathbb{Q}$. We follow the approach of [Sarnak 1983, pages 261-262] in dimension 3. See the appendix for a completely different proof of the same result by V. Emery. 
Theorem 8. Let $\mathbb{O}$ be a maximal order in a definite quaternion algebra A over $\mathbb{Q}$ with discriminant $D_{A}$. Then

$$
\operatorname{Vol}\left(\mathrm{PSL}_{2}(\mathrm{O}) \backslash \mathbb{M}_{\mathbb{R}}^{5}\right)=\frac{\zeta(3) \prod_{p \mid D_{A}}\left(p^{3}-1\right)(p-1)}{11520}
$$

Proof. It is well known (see for instance [Parkkonen and Paulin 2010, Section 6.3, Example (3)]) that there exists $\underline{G}$, a connected semisimple linear algebraic group over $\mathbb{Q}$, such that $\underline{G}(\mathbb{R})=\mathrm{SL}_{2}(\mathbb{H}), \underline{G}(\mathbb{Q})=\mathrm{SL}_{2}(A)$ and $\underline{G}(\mathbb{Z})=\mathrm{SL}_{2}(\mathbb{O})$. Let $\underline{P}$ be the parabolic subgroup of $\underline{G}$, defined over $\mathbb{Q}$, such that $\underline{P}(\mathbb{R})$ is the upper triangular subgroup of $\mathrm{SL}_{2}(\mathbb{M})$. By Borel's finiteness theorem [Borel 1966], the set $\mathrm{SL}_{2}(\mathcal{O}) \backslash \mathrm{SL}_{2}(A) / \underline{P}(\mathbb{Q})$ is finite, and we will fix a subset $\mathscr{R}$ in $\mathrm{SL}_{2}(A)$ which is a system of representatives of this set of double cosets.

Let $\Gamma=\mathrm{SL}_{2}(\mathcal{O})$. For every $\alpha \in \mathscr{R}$, let $\Gamma_{\alpha}=\underline{P}(\mathbb{R}) \cap\left(\alpha^{-1} \Gamma \alpha\right)$ and let $\Gamma_{\alpha}^{\prime}$ be its subgroup of unipotent elements. The group $\alpha \Gamma_{\alpha} \alpha^{-1}$ is the stabilizer of the parabolic

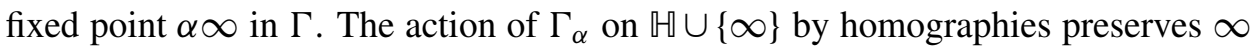
and is cocompact on $\mathbb{H}$. If

$$
\alpha^{-1}=\left(\begin{array}{ll}
a & b \\
c & d
\end{array}\right) \quad \text { and } \quad \alpha=\left(\begin{array}{cc}
\tilde{a} & \tilde{b} \\
\tilde{c} & \tilde{d}
\end{array}\right)
$$

let $\mathfrak{u}_{\alpha}=c \mathcal{O}+d \mathscr{O}$, which is a right fractional ideal of $\mathcal{O}$, and $\mathfrak{v}_{\alpha}=\mathscr{O} \tilde{a}+\mathscr{O} \tilde{c}$, which is a left fractional ideal of $\mathbb{O}$.

For every $\alpha \in \mathscr{R}$, the Eisenstein series of the arithmetic group $\Gamma$ for the cusp at infinity $\alpha \infty$ is the map $\left.E_{\alpha}: \mathbb{U}_{\mathbb{R}}^{5} \times\right] 4,+\infty[\rightarrow \mathbb{R}$ defined by

$$
E_{\alpha}(x, s)=\sum_{\gamma \in\left(\alpha \Gamma_{\alpha} \alpha^{-1}\right) \backslash \Gamma} r\left(\alpha^{-1} \gamma x\right)^{s} .
$$

The summation does not depend on the choice of representatives of the left cosets in $\left(\alpha \Gamma_{\alpha} \alpha^{-1}\right) \backslash \Gamma$ since $\Gamma_{\alpha}$ preserves $\infty$ and the Euclidean height $r$. The Eisenstein series of $\mathcal{O}$ is (for $x=(z, r) \in \mathbb{U}_{\mathbb{R}}^{5}$ and $s \in \mathbb{C}$ with $\operatorname{Re} s>4$ )

$$
\hat{E}(x, s)=\sum_{(c, d) \in \mathcal{O} \times \mathbb{O}-\{0\}}\left(\frac{r}{\mathrm{n}(c z+d)+r^{2} \mathrm{n}(c)}\right)^{s} .
$$

The next result concatenates results proven in [Krafft and Osenberg 1990].

Theorem 9 (Krafft and Osenberg). (i) The Eisenstein series $E_{\alpha}(x, s)$ for $\alpha \in \mathscr{R}$ and $\hat{E}(x, s)$ converge absolutely and uniformly on compact subsets of $\{s \in \mathbb{C}$ : $\operatorname{Res}>4\}$, uniformly on compact subsets of $x \in \mathbb{H}_{\mathbb{R}}^{5}$. They are invariant by the action of $\Gamma$ on the first variable. 
(ii) The map $s \mapsto \hat{E}(x, s)$ admits a meromorphic extension to $\mathbb{C}$, having only one pole, which is at $s=4$ and is simple with residue

$$
\operatorname{Res}_{s=4} \hat{E}(x, s)=\frac{8 \pi^{4}}{3 D_{A}^{2}} .
$$

Furthermore, if $c(\alpha, s)=\mathrm{n}\left(\mathfrak{u}_{\alpha}\right)^{s} \zeta\left(\mathfrak{u}_{\alpha}^{-1}, s / 2\right)$ for every $\alpha \in \mathscr{R}$, then

$$
\hat{E}(x, s)=\sum_{\alpha \in \mathscr{R}} c(\alpha, s) E_{\alpha}(x, s) .
$$

(iii) For every $\alpha, \beta \in \mathscr{R}$, there exists a map $s \mapsto \varphi_{\alpha, \beta}(s)$ with $(s-4) \varphi_{\alpha, \beta}(s)$ bounded for $s>4$ near $s=4$, and a measurable map $(x, s) \mapsto \Phi_{\alpha, \beta}(x, s)$ such that $(s-4) \Phi_{\alpha, \beta}(x, s)$ is bounded by an integrable (for the hyperbolic volume) map, independent on $s>4$ near $s=4$, on $x \in K \times[\epsilon,+\infty[$ where $K$ is a compact subset of $\mathbb{W}$ and $\epsilon>0$, such that

$$
E_{\alpha}(\beta x, s)=\delta_{\alpha, \beta} r^{s}+\varphi_{\alpha, \beta}(s) r^{4-s}+\Phi_{\alpha, \beta}(x, s),
$$

with $\delta_{\alpha, \beta}=1$ if $\alpha=\beta$ and $\delta_{\alpha, \beta}=0$ otherwise.

Proof. We are using Langlands' convention for the Eisenstein series; hence with $\Gamma_{\alpha}^{\prime}$ the subgroup of unipotent elements of $\Gamma_{\alpha}$, our Eisenstein series $E_{\alpha}$ is obtained from the one used in [Krafft and Osenberg 1990] by replacing $\alpha$ by $\alpha^{-1}$ and by multiplying by $1 /\left[\Gamma_{\alpha}: \Gamma_{\alpha}^{\prime}\right]$.

The part of claim (i) concerning the series $E_{\alpha}(x, s)$ for $\alpha \in \mathscr{R}$ is [Krafft and Osenberg 1990, Satz 3.2]. The rest follows from [ibid., Satz 4.2] with $M=0$. The claim (ii) follows from [ibid., Korollar 5.6(a)] with $M=0$, recalling that the reduced discriminant of any maximal order of $A$ is equal to the reduced discriminant of $A$. The formula (19) follows from [ibid., Satz 4.3], recalling the above changes between our $E_{\alpha}$ and the one in [ibid.]. The claim (iii) follows from [ibid., Satz 3.3], again replacing $\beta$ by $\beta^{-1}$, and using the second equation in [Magnus et al. 1966, page 85 ] to control the modified Bessel function.

By a fundamental domain for a smooth action of a countable group $G$ on a smooth manifold $N$, we mean a subset $F$ of $N$ such that $F$ has negligible boundary, the interiors of the subsets $g F$ for $g \in G$ are pairwise disjoint, and

$$
N=\bigcup_{g \in G} g F .
$$

Here is a construction of a fundamental domain $\mathscr{F}$ for $\Gamma$ acting on $\mathbb{T}_{\mathbb{R}}^{5}$ that will be useful in this section (and is valid for any discrete subgroup of isometries of $\mathbb{U}_{\mathbb{R}}^{n}$ with finite covolume which is not cocompact). Let $\mathscr{P}$ be the set of parabolic fixed points of $\Gamma$. By the structure of the cusp neighborhoods, there exists a family $\left(\mathscr{H}_{p}\right)_{p \in \mathscr{P}}$ of pairwise disjoint closed horoballs in $\mathbb{U}_{\mathbb{R}}^{5}$, equivariant under $\Gamma$ (that is, 
$\gamma \mathscr{H}_{p}=\mathscr{H}_{\gamma p}$ for every $\gamma \in \Gamma$ ), with $\mathscr{H}_{p}$ centered at $p$. The cut locus of the cusps $\Sigma$ is the piecewise hyperbolic polyhedral complex in $\mathbb{T}_{\mathbb{R}}^{5}$ consisting of the set of points outside the union of these horoballs that are equidistant to at least two of these horoballs (it is independent of the choice of this family when there is only one orbit of parabolic fixed points). Each connected component of the complement of $\Sigma$ contains one and only one of these horoballs, is at bounded Hausdorff distance of it, is invariant under the stabilizer in $\Gamma$ of its point at infinity, and is precisely invariant under the action of $\Gamma$. Recall that a subset $A$ of a set endowed with an action of a group $G$ is said to be precisely invariant under this group if for every $g \in G$, if $g A \cap A$ is nonempty, then $g A=A$.

For every $\beta \in \mathscr{R}$, let $\mathscr{D}_{\beta}$ be a compact fundamental domain for the action of $\Gamma_{\beta}$ on $\mathbb{H}$, let $\widetilde{\mathscr{F}}_{\beta}$ be the closure of the component of the complement of $\Sigma$ containing $\mathscr{H}_{\beta \infty}$, and define $\mathscr{F}_{\beta}=\widetilde{\mathscr{F}}_{\beta} \cap \beta\left(\mathscr{D}_{\beta} \times\right] 0,+\infty[)$. Then $\mathscr{F}_{\beta}$ is a closed fundamental domain for the action of $\beta \Gamma_{\beta} \beta^{-1}$ on $\widetilde{\mathscr{F}}_{\beta}$, and there exists a continuous map $\left.\sigma_{\beta}^{\prime}: \mathscr{D}_{\beta} \rightarrow\right] 0,+\infty[$, which hence has a positive lower bound, such that

$$
\beta^{-1 \mathscr{F}_{\beta}}=\left\{(z, r) \in \mathbb{T}_{\mathbb{R}}^{5}: z \in \mathscr{D}_{\beta}, r \geq \sigma_{\beta}^{\prime}(z)\right\} .
$$

Now define

$$
\mathscr{F}=\bigcup_{\beta \in \mathscr{R}} \mathscr{F}_{\beta} .
$$

Since $\mathscr{R}$ is a system of representatives of the cusps, $\mathscr{F}$ is a fundamental domain of $\Gamma$ acting on $\mathbb{H}_{\mathbb{R}}^{5}$.

Note that, for every $\alpha \in \mathscr{R}$, there exists a continuous map $\sigma_{\alpha}: \mathscr{D}_{\alpha} \rightarrow[0,+\infty[$ (hence with a finite upper bound), with only finitely many zeros, such that, since $\alpha^{-1} \mathscr{F}$ is a fundamental domain for the action of $\alpha^{-1} \Gamma \alpha$ on $\mathbb{H}_{\mathbb{R}}^{5}$,

$$
\bigcup_{\gamma \in\left(\alpha^{-1} \Gamma \alpha-\Gamma_{\alpha}\right)} \gamma \alpha^{-1} \mathscr{F}=\Gamma_{\alpha}\left\{(z, r) \in \mathbb{M}_{\mathbb{R}}^{5}: z \in \mathscr{D}_{\alpha}, r<\sigma_{\alpha}(z)\right\} .
$$

For every $\alpha \in \mathscr{R}$, let

$$
b_{\alpha}(s)=\int_{\mathscr{F}}\left(E_{\alpha}(x, s)-r\left(\alpha^{-1} x\right)^{s}\right) d \operatorname{vol}_{\mathbb{T}_{\mathbb{R}}^{5}}(x) .
$$

When $s>4$, we have

$b_{\alpha}(s)$

$$
\begin{aligned}
& =\int_{\mathscr{F}}\left(\sum_{\gamma \in\left(\alpha \Gamma_{\alpha} \alpha^{-1}\right) \backslash \Gamma} r\left(\alpha^{-1} \gamma x\right)^{s}-r\left(\alpha^{-1} x\right)^{s}\right) d \operatorname{vol}_{\mathbb{P}_{\mathbb{R}}^{5}}(x) \\
& =\int_{\mathscr{F}} \sum_{\gamma \in} r\left(\gamma \alpha^{-1} x\right)^{s} d \operatorname{vol}_{\mathbb{H}_{\mathbb{R}}^{5}}(x)=\sum_{\gamma \in} \int_{\mathscr{F}} r\left(\gamma \alpha^{-1} x\right)^{s} d \operatorname{vol}_{\mathbb{H}_{\mathbb{R}}^{5}}(x) \\
& \Gamma_{\alpha} \backslash\left(\alpha^{-1} \Gamma \alpha-\Gamma_{\alpha}\right) \\
& \Gamma_{\alpha} \backslash\left(\alpha^{-1} \Gamma \alpha-\Gamma_{\alpha}\right)
\end{aligned}
$$




$$
\begin{aligned}
= & \sum_{\substack{\gamma \in \\
\Gamma_{\alpha} \backslash\left(\alpha^{-1} \Gamma \alpha-\Gamma_{\alpha}\right)}} \int_{\gamma \alpha^{-1} \mathscr{F}} r(x)^{s} d \operatorname{vol}_{\mathbb{F}_{\mathbb{R}}^{5}}(x)=\int_{\bigcup_{\gamma \in \Gamma_{\alpha} \backslash\left(\alpha^{-1} \Gamma \alpha-\Gamma_{\alpha}\right)} \gamma \alpha^{-1 \mathscr{F}}} r(x)^{s} d \operatorname{vol}_{\mathbb{F}_{\mathbb{R}}^{5}}(x) \\
= & \int_{z \in \mathscr{D}_{\alpha}} \int_{0}^{\sigma_{\alpha}(z)} r^{s-5} d r d z=\int_{z \in \mathscr{D}_{\alpha}} \frac{\sigma_{\alpha}(z)^{s-4}}{s-4} d z,
\end{aligned}
$$

using for the succession of equations, respectively, the definition of $E_{\alpha}$, the change of variables $\alpha^{-1} \gamma \alpha \rightarrow \gamma$, Fubini's theorem for positive functions, the invariance of the volume under the isometric change of variables $\gamma \alpha^{-1} x \rightarrow x$, the $\sigma$-additivity property, and the equations (22) and (13) and the invariance of the Euclidean height function $r$ under $\Gamma_{\alpha}$.

For any $\alpha \in \mathscr{R}$, the map $\sigma_{\alpha}{ }^{s-4}$ converges pointwise, as $s \rightarrow 4^{+}$, to the map on $\mathscr{D}_{\alpha}$ with value 0 at the finitely many points where $\sigma_{\alpha}$ vanishes, and with value 1 otherwise. Since $\mathscr{D}_{\alpha}$ is compact and $\sigma_{\alpha}{ }^{s-4}$ is uniformly bounded from above, Lebesgue's dominated convergence theorem gives

$$
\lim _{s \rightarrow 4^{+}}(s-4) b_{\alpha}(s)=\operatorname{Vol}\left(\mathscr{D}_{\alpha}\right)=\operatorname{Vol}\left(\Gamma_{\alpha} \backslash \mathbb{H}\right)
$$

Therefore by using (19), the map

$$
s \mapsto b(s)=\int_{\mathscr{F}}\left(\hat{E}(x, s)-\sum_{\alpha \in \mathscr{R}} c(\alpha, s) r\left(\alpha^{-1} x\right)^{s}\right) d \operatorname{vol}_{\mathbb{R}_{\mathbb{R}}^{5}}(x)=\sum_{\alpha \in \mathscr{R}} c(\alpha, s) b_{\alpha}(s)
$$

satisfies

$$
\lim _{s \rightarrow 4^{+}}(s-4) b(s)=\sum_{\alpha \in \mathscr{R}} c(\alpha, 4) \operatorname{Vol}\left(\Gamma_{\alpha} \backslash \mathbb{H}\right),
$$

since $s \mapsto c(\alpha, s)$ is holomorphic for $\operatorname{Re} s>2$.

On the other hand, let us prove that we may permute the limit as $s \rightarrow 4^{+}$and the integral defining $(s-4) b(s)$. Using the equations (19) and (21), and an isometric, hence volume-preserving, change of variable, we have

$$
\begin{aligned}
b(s) & =\sum_{\alpha, \beta \in \mathscr{R}} c(\alpha, s) \int_{\mathscr{F}_{\beta}}\left(E_{\alpha}(x, s)-r\left(\alpha^{-1} x\right)^{s}\right) d \operatorname{vol}_{\mathbb{T}_{\mathbb{R}}^{5}}(x) \\
& =\sum_{\alpha, \beta \in \mathscr{R}} c(\alpha, s) \int_{\beta^{-1 \mathscr{F}_{\beta}}}\left(E_{\alpha}(\beta x, s)-r\left(\alpha^{-1} \beta x\right)^{s}\right) d \operatorname{vol}_{\mathbb{Q}_{\mathbb{R}}^{5}}(x) .
\end{aligned}
$$

If $x \in \beta^{-1 \mathscr{F}_{\beta}}$, then $r(x)$ is bounded from below by a positive constant by the construction of $\mathscr{F}_{\beta}$; hence $r(x)^{4-s}$ is bounded from above for every $s \geq 4$. If $\alpha \neq \beta$ and $x \in \beta^{-1} \mathscr{F}_{\beta}$, then $r\left(\alpha^{-1} \beta x\right)^{s}$ is bounded from above for every $s \geq 0$, since $\alpha^{-1 \mathscr{F}_{\beta}}$ is bounded in $\mathbb{H} \times \mathbb{R}$ by construction. Hence since $\beta^{-1} \mathscr{F}_{\beta}$ has finite hyperbolic volume, by Theorem 9(iii) separating the case $\alpha=\beta$ and the case $\alpha \neq \beta$, by Lebesgue's dominated convergence theorem, we may permute the 
limit as $s \rightarrow 4^{+}$and the integral on $\beta^{-1} \mathscr{F}_{\beta}$ for the hyperbolic volume applied to $(s-4)\left(E_{\alpha}(\beta x, s)-r\left(\alpha^{-1} \beta x\right)^{s}\right)$. By a finite summation, we may indeed permute the limit as $s \rightarrow 4^{+}$and the integral defining $(s-4) b(s)$.

Therefore, by (18),

$$
\lim _{s \rightarrow 4^{+}}(s-4) b(s)=\frac{8 \pi^{4}}{3 D_{A}^{2}} \operatorname{Vol}\left(\mathrm{PSL}_{2}(\mathcal{O}) \backslash \mathbb{H}_{\mathbb{R}}^{5}\right) .
$$

Finally, since for every $\rho \in A-\{0\}$ the element

$$
\gamma_{\rho}=\left(\begin{array}{cc}
\rho & -1 \\
1 & 0
\end{array}\right)
$$

of $\mathrm{SL}_{2}(A)$ maps $\infty$ to $\rho$, the element $\alpha \in \mathscr{R}$ may be chosen to be either id or $\gamma_{\rho}$ for some $\rho \in A$. In the first case, $\mathfrak{u}_{\alpha}=\mathcal{O}$ and $\Gamma_{\alpha}^{\prime}$ acts on $\mathbb{U}$ as the $\mathbb{Z}$-lattice $\mathfrak{O}$, so that, by (5), since the subgroup $\{ \pm \mathrm{id}\}$ of $\Gamma_{\alpha}$ is the kernel of its action on $\mathbb{H}$,

$$
\mathrm{n}\left(\mathfrak{u}_{\alpha}\right)^{4} \operatorname{Vol}\left(\Gamma_{\alpha} \backslash \mathbb{M}\right)=\frac{2 \operatorname{Vol}(\mathbb{O} \backslash \mathbb{M})}{\left[\Gamma_{\alpha}: \Gamma_{\alpha}^{\prime}\right]}=\frac{D_{A}}{2\left[\Gamma_{\alpha}: \Gamma_{\alpha}^{\prime}\right]} .
$$

In the second case,

$$
\alpha^{-1}=\left(\begin{array}{cc}
0 & 1 \\
-1 & \rho
\end{array}\right)
$$

so that $\mathfrak{u}_{\alpha}=\mathscr{O} \rho+\mathcal{O}$ and $\Gamma_{\alpha}^{\prime}$ acts on $\mathbb{U}$ as the $\mathbb{Z}$-lattice $\Lambda=\mathscr{O} \cap \rho^{-1} \mathcal{O} \cap \mathcal{O} \rho^{-1} \cap \rho^{-1} \mathscr{O} \rho^{-1}$ as we shall see in Lemma 15. By Lemma 6 applied with $z=\rho^{-1}$ and by (5), we hence have

$$
\mathrm{n}\left(\mathfrak{u}_{\alpha}\right)^{4} \operatorname{Vol}\left(\Gamma_{\alpha} \backslash \mathbb{M}\right)=\mathrm{n}\left(\mathfrak{u}_{\alpha}\right)^{4}[\mathbb{O}: \Lambda] \frac{2 \operatorname{Vol}(\mathscr{O} \backslash \mathbb{H})}{\left[\Gamma_{\alpha}: \Gamma_{\alpha}^{\prime}\right]}=\frac{D_{A}}{2\left[\Gamma_{\alpha}: \Gamma_{\alpha}^{\prime}\right]} .
$$

Therefore, by the definition of $c(\alpha, s)$,

$$
\sum_{\alpha \in \mathscr{R}} c(\alpha, 4) \operatorname{Vol}\left(\Gamma_{\alpha} \backslash \mathbb{H}\right)=\frac{D_{A}}{2} \sum_{\alpha \in \mathscr{R}} \frac{\zeta\left(\mathfrak{u}_{\alpha}^{-1}, 2\right)}{\left[\Gamma_{\alpha}: \Gamma_{\alpha}^{\prime}\right]} .
$$

Combining the equations (23), (24) and (25), we have

$$
\operatorname{Vol}\left(\mathrm{PSL}_{2}(\mathrm{O}) \backslash \mathbb{M}_{\mathbb{R}}^{5}\right)=\frac{3 D_{A}^{3}}{16 \pi^{4}} \sum_{\alpha \in \mathscr{R}} \frac{\zeta\left(\mathfrak{u}_{\alpha}^{-1}, 2\right)}{\left[\Gamma_{\alpha}: \Gamma_{\alpha}^{\prime}\right]} .
$$

Lemma 10. (1) For every $\alpha \in \mathscr{R}$, we have $\left[\Gamma_{\alpha}: \Gamma_{\alpha}^{\prime}\right]=\left|\mathcal{O}_{r}\left(\mathfrak{u}_{\alpha}^{-1}\right)^{\times}\right|\left|\mathcal{O}_{r}\left(\mathfrak{v}_{\alpha}\right)^{\times}\right|$.

(2) The map from $\mathscr{R}$ to ${ }_{0} \mathscr{I} \times{ }_{\mathcal{O}} \mathscr{I}$ defined by $\alpha \mapsto\left(\left[\mathfrak{v}_{\alpha}\right],\left[\mathfrak{u}_{\alpha}^{-1}\right]\right)$ is a bijection.

Proof. (1) Let

$$
\Gamma_{\alpha}^{+}=\left\{\gamma \in \Gamma_{\alpha}:\left(\begin{array}{ll}
0 & 1
\end{array}\right) \gamma=\left(\begin{array}{ll}
0 & 1
\end{array}\right)\right\} \quad \text { and } \quad \Gamma_{\alpha}^{-}=\left\{\gamma \in \Gamma_{\alpha}: \gamma\left(\begin{array}{l}
1 \\
0
\end{array}\right)=\left(\begin{array}{l}
1 \\
0
\end{array}\right)\right\},
$$


which are normal subgroups of $\Gamma_{\alpha}$, whose union generates $\Gamma_{\alpha}$. By the top of page 434 in [Krafft and Osenberg 1990] (keeping in mind that our $\alpha$ is the inverse of the $\alpha$ in [ibid.]), we have $\left[\Gamma_{\alpha}^{+}: \Gamma_{\alpha}^{\prime}\right]=\left|\mathcal{O}_{r}\left(\mathfrak{v}_{\alpha}\right)^{\times}\right|$. Similarly, $\left[\Gamma_{\alpha}^{-}: \Gamma_{\alpha}^{\prime}\right]=\left|\mathcal{O}_{\ell}\left(\mathfrak{u}_{\alpha}\right)^{\times}\right|$. Note that $\Gamma_{\alpha}^{\prime}$ is a normal subgroup of $\Gamma_{\alpha}^{-}, \Gamma_{\alpha}^{+}$and $\Gamma_{\alpha}$, such that $\Gamma_{\alpha}^{-} \cap \Gamma_{\alpha}^{+}=\Gamma_{\alpha}^{\prime}$. Hence the product map from $\Gamma_{\alpha}^{-} \times \Gamma_{\alpha}^{+}$to $\Gamma_{\alpha}^{\prime}$ induces a bijection from $\left(\Gamma_{\alpha}^{-} / \Gamma_{\alpha}^{\prime}\right) \times\left(\Gamma_{\alpha}^{\prime} \backslash \Gamma_{\alpha}^{+}\right)$ to $\Gamma_{\alpha} / \Gamma_{\alpha}^{\prime}$, since $\Gamma_{\alpha} / \Gamma_{\alpha}^{\prime}$ is abelian. In particular, $\left[\Gamma_{\alpha}: \Gamma_{\alpha}^{\prime}\right]=\left|\mathcal{O}_{\ell}\left(\mathfrak{u}_{\alpha}\right)^{\times}\right|\left|\mathcal{O}_{r}\left(\mathfrak{v}_{\alpha}\right)^{\times}\right|$. Using (4), the result follows.

(2) Since these matrices act transitively on $A$ by homographies, we may assume that every $\alpha \in \mathscr{R}$ either is the identity element id, or has the form

$$
\left(\begin{array}{cc}
\rho_{\alpha} & -1 \\
1 & 0
\end{array}\right)
$$

for some $\rho_{\alpha} \in A^{\times}$. Then $\alpha^{-1}$ is either id or

$$
\left(\begin{array}{cc}
0 & 1 \\
-1 & \rho_{\alpha}
\end{array}\right)
$$

Hence, $\mathfrak{u}_{\alpha}=\mathbb{O}+\rho_{\alpha} \mathfrak{O}$ and $\mathfrak{v}_{\alpha}=\mathfrak{O} \rho_{\alpha}+\mathfrak{O}$, unless $\alpha=\mathrm{id}$, in which case $\mathfrak{u}_{\alpha}=\mathfrak{v}_{\alpha}=\mathfrak{O}$. Since $\mathrm{SL}_{2}(A)$ acts (on the left) transitively by homographies on $\mathbb{P}_{r}^{1}(\mathbb{O})$ with stabilizer of $[1: 0]$ equal to $\underline{P}(\mathbb{Q})$, the map from $\mathscr{R}$ to $\Gamma_{\mathscr{O}} \backslash \mathbb{P}_{r}^{1}(\mathcal{O})$ defined by $\alpha \mapsto \Gamma_{\mathscr{O}} \alpha[1: 0]$ is a bijection. Note that $\alpha[1: 0]=\left[\rho_{\alpha}: 1\right]$ if $\alpha \neq$ id. Using the notation of Remark 7, if $\alpha \neq \mathrm{id}$, we have $\mathfrak{v}_{\alpha}=I_{\rho_{\alpha}, 1}$ and

$$
\left[K_{\rho_{\alpha}, 1}\right]=\left[\mathcal{O} \rho_{\alpha} \cap \mathcal{O}\right]=\left[\mathcal{O} \cap \mathcal{O} \rho_{\alpha}^{-1}\right]=\left[\mathfrak{u}_{\alpha}^{-1}\right]
$$

by (3). The second assertion of this lemma then follows from Remark 7.

Now, using respectively (9), Lemma 10(1), Lemma 10(2), the separation of variables and (7), (8), and (6) since $\zeta(4)=\pi^{4} / 90$, we have

$$
\begin{aligned}
\sum_{\alpha \in \mathscr{R}} \frac{\zeta\left(\mathfrak{u}_{\alpha}^{-1}, 2\right)}{\left[\Gamma_{\alpha}: \Gamma_{\alpha}^{\prime}\right]} & =\sum_{\alpha \in \mathscr{R}} \frac{\left|\mathcal{O}_{r}\left(\mathfrak{u}_{\alpha}^{-1}\right)^{\times}\right| \zeta_{\left[\mathfrak{u}_{\alpha}^{-1}\right]}(2)}{\left[\Gamma_{\alpha}: \Gamma_{\alpha}^{\prime}\right]}=\sum_{\alpha \in \mathscr{R}} \frac{\zeta_{\left[\mathfrak{u}_{\alpha}^{-1}\right]}(2)}{\left|\mathfrak{O}_{r}\left(\mathfrak{v}_{\alpha}\right)^{\times}\right|} \\
& =\sum_{([I],[J]) \in_{\odot} \mathscr{I}_{\times_{\odot}} \mathscr{I}} \frac{\zeta_{[J]}(2)}{\left|\mathcal{O}_{r}(I)^{\times}\right|}=\zeta_{A}(2) \sum_{[I] \in_{\odot} \mathscr{I}} \frac{1}{\left|\mathcal{O}_{r}(I)^{\times}\right|} \\
& =\frac{\zeta_{A}(2)}{24} \prod_{p \mid D_{A}}(p-1)=\frac{\zeta(3) \pi^{4} \prod_{p \mid D_{A}}\left(1-p^{-3}\right)(p-1)}{2160}
\end{aligned}
$$

Theorem 8 follows from the equations (26) and (27).

Corollary 11. Let $A$ be a definite quaternion algebra over $\mathbb{Q}$ with reduced discriminant $D_{A}$ and class number 1 , and let 0 be a maximal order in $A$. Then the 
hyperbolic volume of $\mathrm{PSL}_{2}(\mathrm{O}) \backslash \mathbb{\mathbb { N } _ { \mathbb { R } } ^ { 5 }}$ is equal to

$$
\operatorname{Vol}\left(\mathrm{PSL}_{2}(\mathcal{O}) \backslash \mathbb{H}_{\mathbb{R}}^{5}\right)=\frac{\left(D_{A}^{3}-1\right)\left(D_{A}-1\right) \zeta(3)}{11520} .
$$

This is an immediate consequence of Theorem 8 . But here is a proof directly from (26) that avoids using the technical Lemma 10 and the technical computation (27).

Proof. Since the number of cusps of $\mathrm{SL}_{2}(\mathrm{O})$ is the square of the class number $h_{A}$ of $A$ (see Remark 7), the set $\mathscr{R}$ has only one element, and we may choose $\mathscr{R}=\{\mathrm{id}\}$.

By definition of the Dieudonné determinant and since every element of $0^{\times}$has norm 1, the stabilizer $\Gamma_{\infty}$ of $\infty$ in $\mathrm{SL}_{2}(\mathrm{O})$ is

$$
\Gamma_{\infty}=\left\{\left(\begin{array}{ll}
a & b \\
0 & d
\end{array}\right): a, d \in \mathbb{O}^{\times}, b \in \mathcal{O}\right\} .
$$

The index in $\Gamma_{\infty}$ of its unipotent subgroup is hence $\left|0^{\times}\right|^{2}$. By the equations (10) and (6), Corollary 11 follows from (26), $\zeta(4)=\pi^{4} / 90,\left|0^{\times}\right|=24 /\left(D_{A}-1\right)$ as seen in (2), and since $D_{A}$ is prime when $h_{A}=1$.

Example 12. Let $A$ be Hamilton's quaternion algebra over $\mathbb{Q}$, which satisfies $D_{A}=2$ and $h_{A}=1$. Let $O$ be Hurwitz's maximal order in A. Applying Corollary 11, we get

$$
\operatorname{Vol}\left(\mathrm{PSL}_{2}(\mathrm{O}) \backslash \mathbb{H}_{\mathbb{R}}^{5}\right)=\frac{7 \zeta(3)}{11520}
$$

exactly four times the minimal volume of a cusped hyperbolic 5-orbifold, as we should because the Hurwitz modular group $\mathrm{PSL}_{2}(\mathrm{O})$ is a subgroup of index 4 in the group of the minimal volume cusped hyperbolic orbifold of dimension 5; see [Hild 2007, page 209; Johnson and Weiss 1999, page 186].

\section{Representing integers by binary Hamiltonian forms}

Let $A$ be a definite quaternion algebra over $\mathbb{Q}$, and let $O$ be a maximal order in $A$.

Let us introduce the general counting function we will study. For every indefinite integral binary Hamiltonian form $f$ over $\mathbb{O}$, for every finite index subgroup $G$ of $\mathrm{SL}_{2}(\mathrm{O})$, for every $x, y$ in 0 not both zero, and for every $s>0$, let

$$
\psi_{f, G, x, y}(s)=\operatorname{Card}_{\mathrm{SU}_{f}(\mathcal{O}) \cap G} \backslash\left\{(u, v) \in G(x, y): \mathrm{n}(\mathcal{O} x+\mathscr{O} y)^{-1}|f(u, v)| \leq s\right\} .
$$

The counting function $\psi_{f, G, x, y}$ depends (besides on $f, G$ ) only on the $G$-orbit of $[x: y]$ in $\mathbb{P}_{r}^{1}(\mathcal{O})$.

Here is the notation for the statement of our main result which follows. Given $(x, y) \in \mathcal{O} \times \mathcal{O}$, let $\Gamma_{\mathscr{O}, x, y}$ and $G_{x, y}$ be the stabilizers of $(x, y)$ for the left linear actions of $\Gamma_{\mathscr{O}}=\mathrm{SL}_{2}(\mathcal{O})$ and $G$, respectively, and let $\mathfrak{u}_{x y^{-1}}$ be the right fractional ideal 
$\mathcal{O}$ if $y=0$ and $\mathcal{O}+x y^{-1} \mathcal{O}$ otherwise. Let $\iota_{G}=1$ if $-\mathrm{id} \in G$, and $\iota_{G}=2$ otherwise. Note that the image of $\mathrm{SU}_{f}(\mathrm{O}) \cap G$ in $\mathrm{PSL}_{2}(\mathbb{M})$ is again an arithmetic group.

Theorem 13. Let $f$ be an integral indefinite binary Hamiltonian form of discriminant $\Delta(f)$ over a maximal order $\mathbb{O}$ of a definite quaternion algebra $A$ over $\mathbb{Q}$. Let $x$ and $y$ be elements in 0 not both zero, and let $G$ be a finite index subgroup of $\Gamma_{0}=\mathrm{SL}_{2}(\mathrm{O})$. Then, as s tends to $+\infty$, we have the equivalence

$$
\psi_{f, G, x, y}(s) \sim \frac{540 \iota_{G}\left[\Gamma_{\mathscr{O}, x, y}: G_{x, y}\right] \operatorname{Covol}\left(\mathrm{SU}_{f}(\mathcal{O}) \cap G\right)}{\pi^{2} \zeta(3)\left|\mathcal{O}_{\ell}\left(\mathfrak{u}_{x y^{-1}}\right)^{\times}\right| \Delta(f)^{2}\left[\Gamma_{\mathscr{O}}: G\right] \prod_{p \mid D_{A}}\left(p^{3}-1\right)\left(1-p^{-1}\right)} s^{4} .
$$

Proof. Let us first recall a geometric result from [Parkkonen and Paulin 2012] that will be used to prove this theorem.

Let $n \geq 2$ and let $\mathbb{U}_{\mathbb{R}}^{n}$ be the upper halfspace model of the real hyperbolic space of dimension $n$, with (constant) sectional curvature -1 . Let $F$ be a finite covolume discrete group of isometries of $\mathbb{Q}_{\mathbb{R}}^{n}$. Let $1 \leq k \leq n-1$ and let $\mathscr{C}$ be a real hyperbolic subspace of dimension $k$ of $\mathbb{T}_{\mathbb{R}}^{n}$, whose stabilizer $F_{\mathscr{C}}$ in $F$ has finite covolume. Let $\mathscr{H}$ be a horoball in $\mathbb{U}_{\mathbb{R}}^{n}$, which is precisely invariant under $F$, with stabilizer $F_{\mathscr{H}}$.

For every $\alpha, \beta \in F$, denote by $\delta_{\alpha, \beta}$ the common perpendicular geodesic arc between $\alpha \mathscr{C}$ and the horosphere $\beta \partial \mathscr{H}$ if it exists, and let $\ell\left(\delta_{\alpha, \beta}\right)$ be its length, counted positively if $\delta_{\alpha, \beta}$ exits $\beta \mathscr{H}$ at its endpoint on $\beta \partial \mathscr{H}$, and negatively otherwise. Also define the multiplicity of $\delta_{\alpha, \beta}$ as $m(\alpha, \beta)=1 / \operatorname{Card}\left(\alpha F_{\mathscr{C}} \alpha^{-1} \cap \beta F_{\mathscr{H}} \beta^{-1}\right)$. Its denominator is finite, if the boundary at infinity of $\alpha \mathscr{C}$ does not contain the point at infinity of $\beta \mathscr{H}$, since then the subgroup $\alpha F_{\mathscr{C}} \alpha^{-1} \cap \beta F_{\mathscr{H}} \beta^{-1}$ that preserves both $\beta \mathscr{H}$ and $\alpha \mathscr{C}$ consists of elliptic elements. By convention, $\ell\left(\delta_{\alpha, \beta}\right)=-\infty$ and $m(\alpha, \beta)=0$ if the boundary at infinity of $\alpha \mathscr{C}$ contains the point at infinity of $\beta \mathscr{H}$. In particular, there are only finitely many elements $[g] \in F_{\mathscr{C}} \backslash F / F_{\mathscr{H}}$ such that $m\left(g^{-1}\right.$, id) is different from 1 , or equivalently such that $g^{-1} F_{\mathscr{C}} g \cap F_{\mathscr{H}} \neq\{1\}$. For every $t \geq 0$, define $\mathcal{N}(t)=\mathcal{N}_{F, \mathscr{C}, \mathscr{H}}(t)$ as the number, counted with multiplicity, of the orbits under $F$ in the set of the common perpendicular $\operatorname{arcs} \delta_{\alpha, \beta}$ for $\alpha, \beta \in F$ with length at most $t$ :

$$
\mathcal{N}(t)=\mathcal{N}_{F, \mathscr{C}, \mathscr{H}}(t)=\sum_{\substack{(\alpha, \beta) \in F \backslash\left(\left(F / F_{\mathscr{G}}\right) \times\left(F / F_{\mathscr{H}}\right)\right) \\ \ell\left(\delta_{\alpha, \beta}\right) \leq t}} m(\alpha, \beta) .
$$

For every $m \in \mathbb{N}$, denoting by $\mathbb{S}_{m}$ the unit sphere of the Euclidean space $\mathbb{R}^{m+1}$ endowed with its induced Riemannian metric, we have the following result:

Theorem 14 [Parkkonen and Paulin 2012, Corollary 4.9]. As $t \rightarrow+\infty$, we have

$$
\mathcal{N}(t) \sim \frac{\operatorname{Vol}\left(\mathbb{S}_{n-k-1}\right) \operatorname{Vol}\left(F_{\mathscr{H}} \backslash \mathscr{H}\right) \operatorname{Vol}\left(F_{\mathscr{C}} \backslash \mathscr{C}\right)}{\operatorname{Vol}\left(\mathbb{S}_{n-1}\right) \operatorname{Vol}\left(F \backslash \mathbb{G} \mathbb{R}_{\mathbb{R}}^{n}\right)} e^{(n-1) t} .
$$


Now, let $A, \mathcal{O}, f, G, x$ and $y$ be as in the statement of Theorem 13. We write $f$ as in (1), and denote its discriminant by $\Delta$. In order to apply Theorem 14, we first define the various objects $n, k, F$, $\mathscr{H}$, and $\mathscr{C}$ that appear in its statement.

Let $n=5$ and $k=4$, so that $\operatorname{Vol}\left(\mathbb{S}_{n-1}\right)=8 \pi^{2} / 3$ and $\operatorname{Vol}\left(\mathbb{S}_{n-k-1}\right)=2$. We use the description of $\mathbb{W}_{\mathbb{R}}^{5}$ given in Section 3 .

For any subgroup $S$ of $\mathrm{SL}_{2}(\mathbb{M})$, we denote by $\bar{S}$ its image in $\mathrm{PSL}_{2}(\mathbb{M})$, except that the image of $\operatorname{SU}_{f}(\mathcal{O})$ is denoted by $\operatorname{PSU}_{f}(\mathcal{O})$. We will apply Theorem 14 to $F=\bar{G}$.

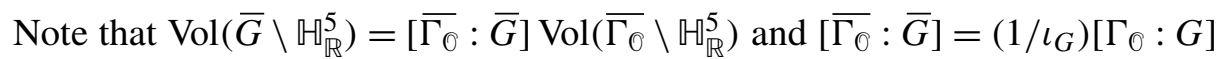
by the definition of $\iota_{G}$. Thus, using Theorem 8 (or Theorem A.1), we have

$$
\begin{aligned}
& \operatorname{Vol}\left(\bar{G} \backslash \mathbb{M}_{\mathbb{R}}^{5}\right)=\frac{1}{\iota_{G}}\left[\Gamma_{\mathscr{O}}: G\right] \operatorname{Vol}\left(\overline{\Gamma_{\overparen{O}}} \backslash \mathbb{M}_{\mathbb{R}}^{5}\right)=\frac{1}{\iota_{G}}\left[\Gamma_{\mathscr{O}}: G\right] \operatorname{Covol}\left(\Gamma_{\mathscr{C}}\right) \\
& =\frac{\zeta(3)\left[\Gamma_{\mathscr{O}}: G\right]}{11520 \iota_{G}} \prod_{p \mid D_{A}}\left(p^{3}-1\right)(p-1) .
\end{aligned}
$$

The point $\rho=x y^{-1} \in A \cup\{\infty\} \subset \partial_{\infty} \mathbb{H}_{\mathbb{R}}^{5}$ is a parabolic fixed point of $\overline{\Gamma_{\overparen{O}}}$ and hence of $\bar{G}$. Let $\tau \in] 0,1]$ and $\mathscr{H}$ be the horoball in $\mathbb{H}_{\mathbb{R}}^{5}$ centered at $\rho$, with Euclidean height $\tau$ if $y \neq 0$, and consisting of the points of Euclidean height at least $1 / \tau$ otherwise. Assume that $\tau$ is small enough so that $\mathscr{H}$ is precisely invariant under $\overline{\Gamma_{\mathscr{O}}}$ and hence under $\bar{G}$. Such a $\tau$ exists, as seen in the construction of the fundamental domain in Section 5. The stabilizer $\overline{\Gamma_{\mathscr{O}, \rho}}$ in $\overline{\Gamma_{\overparen{O}}}$ of the point at infinity $\rho$ is equal to the stabilizer $\left(\overline{\Gamma_{\overparen{O}}}\right) \mathscr{H}$ of the horoball $\mathscr{H}$.

Remark. If $\rho=\infty$ and $G=\Gamma_{0}$, we may take $\tau=1$ by [Kellerhals 2003, Proposition 5]. Then by an easy hyperbolic geometry computation, since the index in $\left(\overline{\Gamma_{\overparen{O}}}\right) \mathscr{H}$ of the subgroup of translations by elements of $\mathcal{O}$ is $\frac{\left|\mathcal{O}^{\times}\right|^{2}}{2}$, and by using (5), we have

$$
\operatorname{Vol}\left(\left(\overline{\Gamma_{\overparen{O}}}\right) \mathscr{H} \backslash \mathscr{H}\right)=\frac{1}{4} \operatorname{Vol}\left(\left(\overline{\Gamma_{\overparen{O}}}\right) \mathscr{H} \backslash \partial \mathscr{H}\right)=\frac{1}{2\left|\mathcal{O}^{\times}\right|^{2}} \operatorname{Vol}(\mathcal{O} \backslash \mathbb{M})=\frac{D_{A}}{8\left|\mathcal{O}^{\times}\right|^{2}}
$$

The following lemma will allow us to generalize this formula.

Lemma 15. Let $\Lambda_{\odot, \rho}^{\prime}=\mathscr{O} \cap \rho^{-1} \mathscr{O} \cap \mathcal{O} \rho^{-1} \cap \rho^{-1} \mathcal{O} \rho^{-1}$ if $x, y \neq 0$, and $\Lambda_{\mathscr{O}, \rho}^{\prime}=0$ otherwise. Then $\Lambda_{\odot, \rho}^{\prime}$ is a $\mathbb{Z}$-lattice in $\mathbb{H}$ and we have

$$
\operatorname{Vol}\left(\bar{G}_{\mathscr{H}} \backslash \mathscr{H}\right)=\frac{\tau^{4}\left[\left(\overline{\Gamma_{\overparen{O}}}\right)_{\mathscr{H}}: \bar{G}_{\mathscr{H}}\right]}{4\left|\mathcal{O}_{\ell}\left(\mathfrak{u}_{\rho}\right)^{\times}\right|\left[\left(\overline{\Gamma_{\mathscr{O}}}\right)_{\mathscr{H}}: \overline{\Gamma_{\mathscr{O}, x, y}}\right]} \operatorname{Vol}\left(\Lambda_{\mathscr{O}, \rho}^{\prime} \backslash \mathbb{H}\right) .
$$

Proof. If $y=0$, let $\gamma_{\rho}=\mathrm{id}$; otherwise let

$$
\gamma_{\rho}=\left(\begin{array}{cc}
\rho & -1 \\
1 & 0
\end{array}\right) \in \mathrm{SL}_{2}(\mathbb{H})
$$


Note that $\gamma_{\rho}^{-1}$ maps $\rho$ to $\infty$ and $\mathscr{H}$ to the horoball $\mathscr{H}_{\infty}$ consisting of the points in $\mathbb{M}_{\mathbb{R}}^{5}$ with Euclidean height at least $1 / \tau$.

Let

$$
\gamma=\left(\begin{array}{ll}
a & b \\
c & d
\end{array}\right) \quad \text { and } \quad \gamma^{\prime}=\left(\begin{array}{ll}
1 & b^{\prime} \\
0 & 1
\end{array}\right)
$$

be in $\mathrm{SL}_{2}(\mathbb{M})$. If $y=0$, we have $\gamma_{\rho}^{-1} \gamma \gamma_{\rho}=\gamma^{\prime}$ if and only if $a=1, b=b^{\prime}, c=0$, $d=1$. If $y \neq 0$, by an easy computation, we have $\gamma_{\rho}^{-1} \gamma \gamma_{\rho}=\gamma^{\prime}$ (that is, $\gamma \gamma_{\rho}=\gamma_{\rho} \gamma^{\prime}$ ) if and only if

$$
c=-b^{\prime}, \quad a=1-\rho b^{\prime}, \quad d=1+b^{\prime} \rho, \quad b=\rho b^{\prime} \rho .
$$

In particular, if $x, y \neq 0$, if $\gamma \in \mathrm{SL}_{2}(0)$ and $\gamma^{\prime}=\gamma_{\rho}^{-1} \gamma \gamma_{\rho} \in \mathrm{SL}_{2}(A)$ is unipotent upper triangular, then these equations imply respectively that $b^{\prime}$ belongs to $\mathbb{O}$, $\rho^{-1} \mathcal{O}, \mathcal{O} \rho^{-1}$ and $\rho^{-1} \mathscr{O} \rho^{-1}$; therefore $b^{\prime} \in \Lambda_{\overparen{O}, \rho}^{\prime}$. If $x=0$ or $y=0$, we also have $b^{\prime} \in \mathbb{O}=\Lambda_{\mathscr{O}, \rho}^{\prime}$

Conversely, if $b^{\prime} \in \Lambda_{\mathscr{O}, \rho}^{\prime}$, then define $a, b, c, d$ by the equations (30) if $y \neq 0$, and by $a=1, b=b^{\prime}, c=0, d=1$ otherwise, so that $a, b, c, d \in \mathbb{O}$. Let

$$
\gamma=\left(\begin{array}{ll}
a & b \\
c & d
\end{array}\right)
$$

If $y \neq 0$, note that if $c=0$, then $\gamma=$ id and otherwise $c b-c a c^{-1} d=-1$, so that $\gamma \in \mathrm{SL}_{2}(0)$ by (12). If $y \neq 0$, the equations (30) imply that $\gamma_{\rho}^{-1} \gamma \gamma_{\rho}$ is a unipotent upper triangular element of $\mathrm{SL}_{2}(0)$, and this is also the case if $y=0$.

The abelian group $\Lambda_{\mathscr{C}, \rho}^{\prime}$ is a $\mathbb{Z}$-lattice in $\mathbb{Z}$, as an intersection of at most four $\mathbb{Z}$-lattices in $A$. Since an isometry preserves the volume for the first equality, by an easy hyperbolic volume computation for the second one, and by the previous computation of the unipotent upper triangular subgroup $\Gamma_{\gamma_{\rho}}^{\prime}$ of $\gamma_{\rho}^{-1} \Gamma_{\mathscr{O}, x, y} \gamma_{\rho}$ for the last one, we have

$$
\begin{aligned}
\operatorname{Vol}\left(\overline{\Gamma_{\mathscr{O}, x, y}} \backslash \mathscr{H}\right) & =\operatorname{Vol}\left(\left(\gamma_{\rho}^{-1} \overline{\Gamma_{\mathscr{O}, x, y}} \gamma_{\rho}\right) \backslash \mathscr{H}_{\infty}\right)=\frac{1}{4} \operatorname{Vol}\left(\left(\gamma_{\rho}^{-1} \overline{\Gamma_{\mathscr{O}, x, y}} \gamma_{\rho}\right) \backslash \partial \mathscr{H}_{\infty}\right) \\
& =\frac{\tau^{4}}{4} \operatorname{Vol}\left(\left(\gamma_{\rho}^{-1} \overline{\Gamma_{\mathscr{O}, x, y}} \gamma_{\rho}\right) \backslash \mathbb{U}\right)=\frac{\tau^{4}}{4\left[\gamma_{\rho}^{-1} \Gamma_{\mathscr{C}, x, y} \gamma_{\rho}: \Gamma_{\gamma_{\rho}}^{\prime}\right]} \operatorname{Vol}\left(\Lambda_{\mathscr{O}, \rho}^{\prime} \backslash \mathbb{U}\right) .
\end{aligned}
$$

With the notation of the proof of Lemma $10(1)$, we have $\left[\gamma_{\rho}^{-1} \Gamma_{\mathscr{O}, x, y} \gamma_{\rho}: \Gamma_{\gamma_{\rho}}^{\prime}\right]=$ $\left|O_{\ell}\left(\mathfrak{u}_{\rho}\right)^{\times}\right|$. Since covering arguments yield

$$
\operatorname{Vol}\left(\bar{G}_{\mathscr{H}} \backslash \mathscr{H}\right)=\left[\left(\overline{\Gamma_{\overparen{O}}}\right) \mathscr{H}: \bar{G}_{\mathscr{H}}\right] \operatorname{Vol}\left(\left(\overline{\Gamma_{\overparen{C}}}\right)_{\mathscr{H}} \backslash \mathscr{H}\right)=\frac{\left[\left(\overline{\Gamma_{\overparen{C}}}\right)_{\mathscr{H}}: \bar{G}_{\mathscr{H}}\right]}{\left[\left(\overline{\Gamma_{\overparen{C}}}\right)_{\mathscr{H}}: \overline{\Gamma_{\overparen{C}, x, y}}\right]} \operatorname{Vol}\left(\overline{\Gamma_{\overparen{C}, x, y}} \backslash \mathscr{H}\right),
$$

the result follows. 
Let us resume the proof of Theorem 13. Let $\mathscr{C}=\mathscr{C}(f)$, which is indeed a real hyperbolic hyperplane in $\mathbb{H}_{\mathbb{R}}^{5}$, whose set of points at infinity is $\mathscr{C}_{\infty}(f)$ (hence $\infty$ is a point at infinity of $\mathscr{C}(f)$ if and only if the first coefficient $a=a(f)$ of $f$ is 0 ). Note that $\mathscr{C}$ is invariant under the group $\mathrm{SU}_{f}(\mathcal{O})$ by (17) (this equation implies that $\mathscr{C}(f \circ g)=g^{-1} \mathscr{C}(f)$ for every $\left.g \in \mathrm{SL}_{2}(\mathcal{O})\right)$. The arithmetic group $\mathrm{SU}_{f}(\mathcal{O})$ acts with finite covolume on $\mathscr{C}(f)$, its finite subgroup $\{ \pm \mathrm{id}\}$ acting trivially. By definition,

$$
\operatorname{Covol}\left(\mathrm{SU}_{f}(\mathbb{O}) \cap G\right)=\operatorname{Vol}\left(\operatorname{PSU}_{f}(\mathbb{O}) \cap \bar{G}^{\backslash \mathscr{C}(f)}\right) .
$$

Note that $\operatorname{Covol}\left(\mathrm{SU}_{f}(\mathcal{O}) \cap G\right)$ depends only on the $G$-orbit of $f$, by (17) and since $\mathrm{SU}_{f \circ g}(\mathcal{O})=g^{-1} \mathrm{SU}_{f}(\mathcal{O}) g$ for every $g \in \mathrm{SL}_{2}(\mathcal{O})$. By its definition, $R_{G}(f)$ is the index of the subgroup $\operatorname{PSU}_{f}(\mathcal{O}) \cap \bar{G}$ in $\bar{G}_{\mathscr{b}}$; hence

$$
\operatorname{Vol}\left(\bar{G}_{\mathscr{C}} \backslash \mathscr{C}\right)=\frac{1}{R_{G}(f)} \operatorname{Covol}\left(\mathrm{SU}_{f}(\mathcal{O}) \cap G\right)
$$

The last step of the proof of Theorem 13 consists in relating the two counting functions $\psi_{f, G, x, y}$ and $\mathcal{N}_{\bar{G}, \ell, \mathscr{H}}$, in order to apply Theorem 14 .

For every $g \in \mathrm{SL}_{2}(\mathbb{H})$, let us compute the hyperbolic length of the common perpendicular geodesic arc $\delta_{g^{-1} \text {,id }}$ between the real hyperbolic hyperplane $g^{-1} \mathscr{C}$ and the horoball $\mathscr{H}$, assuming that they do not meet. We use the notation $\gamma_{\rho}, \mathscr{H}_{\infty}$ introduced in the proof of Lemma 15. Since $\gamma_{\rho}^{-1}$ sends the horoball $\mathscr{H}$ to the horoball $\mathscr{H}_{\infty}$, it sends the common perpendicular geodesic arc between $g^{-1} \mathscr{C}$ and $\mathscr{H}$ to the (vertical) common perpendicular geodesic arc between $\gamma_{\rho}^{-1} g^{-1} \mathscr{C}$ and $\mathscr{H}_{\infty}$. Let $r$ be the Euclidean radius of the 3 -sphere $\mathscr{C}_{\infty}\left(f \circ g \circ \gamma_{\rho}\right)$, which is the image by $\gamma_{\rho}^{-1}$ of the boundary at infinity of $g^{-1} \mathscr{C}$ by (17). Denoting by $a\left(f \circ g \circ \gamma_{\rho}\right)$ the coefficient of $\mathrm{n}(u)$ in $f \circ g \circ \gamma_{\rho}(u, v)$, we have, by (16),

$$
\begin{aligned}
r=\frac{\sqrt{\Delta\left(f \circ g \circ \gamma_{\rho}\right)}}{\left|a\left(f \circ g \circ \gamma_{\rho}\right)\right|} & =\frac{\sqrt{\Delta}}{\left|f \circ g \circ \gamma_{\rho}(1,0)\right|} \\
& =\frac{\sqrt{\Delta}}{|f \circ g(\rho, 1)|}=\frac{\mathrm{n}(y) \sqrt{\Delta}}{|f \circ g(x, y)|},
\end{aligned}
$$

if $y \neq 0$ and $r=(\mathrm{n}(x) \sqrt{\Delta}) /|f \circ g(x, y)|$ otherwise. An immediate computation gives

$$
\ell\left(\delta_{g^{-1}, \mathrm{id}}\right)=\ell\left(\gamma_{\rho}^{-1} \delta_{g^{-1}, \mathrm{id}}\right)=\ln \frac{1}{\tau}-\ln r=\ln \frac{|f \circ g(x, y)|}{\tau \mathrm{n}(y) \sqrt{\Delta}},
$$

if $y \neq 0$ and

$$
\ell\left(\delta_{g^{-1}, \text { id }}\right)=\ln \frac{|f \circ g(x, y)|}{\tau \operatorname{nn}(x) \sqrt{\Delta}}
$$

otherwise. With the conventions that we have taken, these formulas are also valid if $g^{-1} \mathscr{C}$ and $\mathscr{H}$ meet. 
Recall that there are only finitely many elements $[g] \in \bar{G}_{\mathscr{C}} \backslash \bar{G} / \bar{G}_{\mathscr{C}}$ such that $g^{-1} \bar{G}_{\mathscr{C}} g \cap \bar{G}_{\mathscr{H}}$ is different from $\{1\}$ or such that the multiplicity $m\left(g^{-1}\right.$, id) is different from 1. If $y \neq 0$, using (32) for the third line below, [Parkkonen and Paulin 2011, Lemma 7] for the fourth one, and Theorem 14 applied to $F=\bar{G}$ for the sixth one, we hence have, as $s$ tends to $+\infty$,

$\psi_{f, G, x, y}(s)$

$$
\begin{aligned}
& =\operatorname{Card}\left\{[g] \in\left(\mathrm{SU}_{f}(\mathcal{O}) \cap G\right) \backslash G / G_{x, y}: \mathrm{n}(\mathcal{O} x+\mathscr{O} y)^{-1}|f \circ g(x, y)| \leq s\right\} \\
& =\operatorname{Card}\left\{[g] \in\left(\mathrm{PSU}_{f}(\mathcal{O}) \cap \bar{G}\right) \backslash \bar{G} / \overline{G_{x, y}}: \ell\left(\delta_{g^{-1}, \mathrm{id}}\right) \leq \ln \frac{s \mathrm{n}(\mathcal{O} x+\mathcal{O} y)}{\tau \mathrm{n}(y) \sqrt{\Delta}}\right\} \\
& \sim R_{G}(f)\left[\bar{G}_{\mathscr{H}}:{\overline{G_{x, y}}}\right] \operatorname{Card}\left\{[g] \in \bar{G}_{\mathscr{C}} \backslash \bar{G} / \bar{G}_{\mathscr{H}}: \ell\left(\delta_{g^{-1}, \mathrm{id}}\right) \leq \ln \frac{s \mathrm{n}(\mathcal{O} \rho+\mathcal{O})}{\tau \sqrt{\Delta}}\right\} \\
& \sim R_{G}(f)\left[\bar{G}_{\mathscr{H}}: \bar{G}_{x, y}\right] \mathcal{N}_{\bar{G}, \mathscr{C}, \mathscr{H}}\left(\ln \frac{s \mathrm{n}(\mathcal{O} \rho+\mathcal{O})}{\tau \sqrt{\Delta}}\right) \\
& \sim R_{G}(f)\left[\bar{G}_{\mathscr{H}}:{\overline{G_{x, y}}}\right] \frac{6 \operatorname{Vol}\left(\bar{G}_{\mathscr{H}} \backslash \mathscr{H}\right) \operatorname{Vol}\left(\bar{G}_{\mathscr{C}} \backslash \mathscr{C}\right)}{8 \pi^{2} \operatorname{Vol}\left(\bar{G} \backslash \mathbb{U}_{\mathbb{R}}^{n}\right)}\left(\frac{s \mathrm{n}(\mathcal{O} \rho+\mathcal{O})}{\tau \sqrt{\Delta}}\right)^{4} .
\end{aligned}
$$

We replace the three volumes in the computation above by their expressions given in the equations (28), (29) and (31). We simplify the obtained expression using the following two remarks. Firstly,

$\left[\bar{G}_{\mathscr{H}}: \overline{G_{x, y}}\right] \frac{\left[\left(\overline{\Gamma_{\mathscr{O}}}\right)_{\mathscr{H}}: \bar{G}_{\mathscr{H}}\right]}{\left[\left(\overline{\Gamma_{\mathscr{O}}}\right)_{\mathscr{H}}: \overline{\Gamma_{\mathscr{C}, x, y}}\right]}=\frac{\left[\left(\overline{\Gamma_{\overparen{O}}}\right)_{\mathscr{H}}: \overline{G_{x, y}}\right]}{\left[\left(\overline{\Gamma_{\overparen{C}}}\right)_{\mathscr{H}}: \overline{\Gamma_{\mathscr{O}, x, y}}\right]}=\left[\overline{\Gamma_{\mathscr{C}, x, y}}: \overline{G_{x, y}}\right]=\left[\Gamma_{\mathscr{O}, x, y}: G_{x, y}\right]$.

Secondly, we claim that

$$
\operatorname{Vol}\left(\Lambda_{\mathscr{C}, \rho}^{\prime} \backslash \mathbb{M}\right) \mathrm{n}(\mathcal{O} \rho+\mathscr{O})^{4}=\frac{D_{A}}{4} .
$$

If $x=0$, then $\Lambda_{\mathscr{O}, \rho}^{\prime}=0$; hence this claim is true, by (5) and since $\mathrm{n}(\mathcal{O})=1$. Otherwise, claim (33) follows from Lemma 6 with $z=\rho^{-1}$, since, by the definition of $\Lambda_{\sigma, \rho}^{\prime}$,

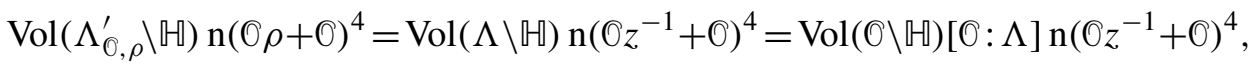
and by (5).

This concludes the proof of Theorem 13 if $y \neq 0$. The case $y=0$ is similar to the case $x=0$.

Let us give a few corollaries of Theorem 13. The first one below follows by taking $G=\mathrm{SL}_{2}(\mathrm{O})$ in Theorem 13 .

Corollary 16. Let $f$ be an integral indefinite binary Hamiltonian form of discriminant $\Delta(f)$ over a maximal order $\mathbb{O}$ of a definite quaternion algebra $A$ over $\mathbb{Q}$. 
Let $x$ and $y$ be elements in 0 not both zero. Then, as $s$ tends to $+\infty$, we have the equivalence

$$
\psi_{f, \mathrm{SL}_{2}(\mathcal{O}), x, y}(s) \sim \frac{540 \operatorname{Covol}\left(\mathrm{SU}_{f}(\mathcal{O})\right)}{\pi^{2} \zeta(3)\left|\mathscr{O}_{\ell}\left(\mathfrak{u}_{x y^{-1}}\right)^{\times}\right| \Delta(f)^{2} \prod_{p \mid D_{A}}\left(p^{3}-1\right)\left(1-p^{-1}\right)} s^{4} .
$$

Remark 17. Recall that by Remark 7, the map from $\mathrm{SL}_{2}(\mathbb{O}) \backslash \mathbb{P}_{r}^{1}(\mathbb{O})$ to $\mathbb{O}^{\mathscr{I}} \times{ }_{\mathbb{O}} \mathscr{I}$ that associates, to the orbit of $[u: v]$ in $\mathbb{P}_{r}^{1}(\mathcal{O})$ under $\mathrm{SL}_{2}(\mathcal{O})$, the couple of ideal classes $\left(\left[I_{u, v}\right],\left[K_{u, v}\right]\right)$ is a bijection. The counting function $\psi_{f, \mathrm{SL}_{2}(\mathcal{O}), x, y}$ hence depends only on $\left(\left[I_{x, y}\right],\left[K_{x, y}\right]\right)$.

Given two left fractional ideals $\mathfrak{m}$ and $\mathfrak{m}^{\prime}$ of $\mathfrak{O}$, let $\psi_{f, \mathfrak{m}, \mathfrak{m}^{\prime}}(s)$ be the cardinality of the set

$$
\mathrm{SU}_{f}(\mathcal{O}) \backslash\left\{(u, v) \in \mathfrak{m} \times \mathfrak{m}: \frac{|f(u, v)|}{\mathrm{n}(\mathfrak{m})} \leq s, I_{u, v}=\mathfrak{m},\left[K_{u, v}\right]=\left[\mathfrak{m}^{\prime}\right]\right\} .
$$

Note that this counting function depends only on the ideal classes of $\mathfrak{m}$ and $\mathfrak{m}^{\prime}$.

Corollary 18. Let $f$ be an integral indefinite binary Hamiltonian form of discriminant $\Delta(f)$ over a maximal order $\mathbb{O}$ of a definite quaternion algebra $A$ over $\mathbb{Q}$. Let $\mathfrak{m}$ and $\mathfrak{m}^{\prime}$ be two left fractional ideals in 0 . Then as $s$ tends to $+\infty$, we have the equivalence

$$
\psi_{f, \mathfrak{m}, \mathfrak{m}^{\prime}}(s) \sim \frac{540 \operatorname{Covol}\left(\mathrm{SU}_{f}(\mathcal{O})\right)}{\pi^{2} \zeta(3)\left|\mathcal{O}_{r}\left(\mathfrak{m}^{\prime}\right)^{\times}\right| \Delta(f)^{2} \prod_{p \mid D_{A}}\left(p^{3}-1\right)\left(1-p^{-1}\right)} s^{4} .
$$

Proof. By Remark 17, we have

$$
\psi_{f, \mathfrak{m}, \mathfrak{m}^{\prime}}=\psi_{f, \mathrm{SL}_{2}(\mathcal{O}), x, y},
$$

where $(x, y)$ is any nonzero element of $\mathbb{O} \times \mathcal{O}$ such that $\left[I_{x, y}\right]=[\mathfrak{m}]$ and $\left[K_{x, y}\right]=\left[\mathfrak{m}^{\prime}\right]$. By the equations (4) and (3), if $x y \neq 0$, we have

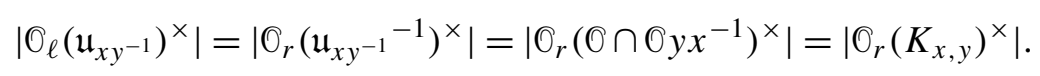

The first and last terms are also equal if $x y=0$. Hence the result follows from Corollary 16.

Remark 19. With $\psi_{f, \mathfrak{m}}$ the counting function defined in the introduction, we have

$$
\psi_{f, \mathfrak{m}}=\sum_{\left[\mathfrak{m}^{\prime}\right] \in_{\Theta^{\mathscr{I}}}} \psi_{f, \mathfrak{m}, \mathfrak{m}^{\prime}}
$$

Therefore, since

$$
\sum_{\left[\mathfrak{m}^{\prime}\right] \in_{\Theta} \mathscr{\Phi}} \frac{1}{\left|\mathcal{O}_{r}\left(\mathfrak{m}^{\prime}\right)^{\times}\right|}=\frac{1}{24} \prod_{p \mid D_{A}}(p-1)
$$

by (8), Theorem 1 in the introduction follows from Corollary 18. 
We say $u, v \in \mathbb{O} \times \mathbb{O}$ are relatively prime if one of the following equivalent (by Remark 17) conditions is satisfied:

(i) There exists $g \in \mathrm{SL}_{2}(0)$ such that $g(1,0)=(u, v)$.

(ii) There exists $u^{\prime}, v^{\prime} \in \mathbb{O}$ such that $\mathrm{n}\left(u v^{\prime}\right)+\mathrm{n}\left(u^{\prime} v\right)-\operatorname{tr}\left(u \bar{v} v^{\prime} \overline{u^{\prime}}\right)=1$.

(iii) The $\mathrm{O}$-modules $I_{u, v}$ and $K_{u, v}$ are isomorphic (as $\mathbf{O}$-modules) to $\mathbb{O}$.

We denote by $\mathscr{P}_{\mathscr{O}}$ the set of couples of relatively prime elements of $\mathcal{O}$.

Corollary 20. Let $f$ be an integral indefinite binary Hamiltonian form over a maximal order $\mathbb{O}$ in a definite quaternion algebra $A$ over $\mathbb{Q}$, and let $G$ be a finite index subgroup of $\Gamma_{\mathbb{O}}=\mathrm{SL}_{2}\left(O_{)}\right.$. Then, as s tends to $+\infty$, we have the equivalence

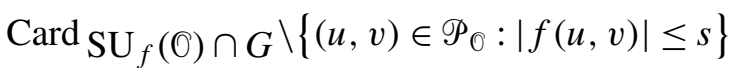

$$
\sim \frac{540 \iota_{G}\left[\Gamma_{\mathscr{O}, 1,0}: G_{1,0}\right] \operatorname{Covol}\left(\mathrm{SU}_{f}(\mathcal{O}) \cap G\right)}{\pi^{2} \zeta(3)\left|\mathcal{O}^{\times}\right| \Delta(f)^{2}\left[\Gamma_{\mathscr{O}}: G\right] \prod_{d \mid D_{A}}\left(p^{3}-1\right)\left(1-p^{-1}\right)} s^{4} .
$$

Proof. This follows from Theorem 13 by taking $x=1$ and $y=0$.

Proof of Corollary 2 from the introduction. Consider the integral indefinite binary Hamiltonian form $f$ over 0 defined by $f(u, v)=\operatorname{tr}(\bar{u} v)$, with matrix

$$
M(f)=\left(\begin{array}{ll}
0 & 1 \\
1 & 0
\end{array}\right)
$$

and discriminant $\Delta(f)=1$. Its group of automorphs is

$$
\mathrm{Sp}_{1}(\mathcal{O})=\left\{g \in \mathrm{SL}_{2}(\mathcal{O}): g^{*}\left(\begin{array}{ll}
0 & 1 \\
1 & 0
\end{array}\right) g=\left(\begin{array}{ll}
0 & 1 \\
1 & 0
\end{array}\right)\right\},
$$

which is an arithmetic lattice in the symplectic group over the quaternions $\operatorname{Sp}_{1}(\mathbb{W})$. We have

$$
\mathscr{C}(f)=\{(z, r) \in \mathbb{H} \times] 0,+\infty[: \operatorname{tr}(z)=0\} .
$$

The hyperbolic volume of the quotient of $\{(z, r) \in \mathbb{U} \times] 0,+\infty[: \operatorname{tr}(z)=0\}$ by $\mathrm{Sp}_{1}(\mathbb{O})$ has been computed as the main result of [Breulmann and Helmke 1996], yielding

$$
\operatorname{Covol}\left(\operatorname{Sp}_{1}(\mathcal{O})\right)=\frac{\pi^{2}}{1080} \prod_{p \mid D_{A}}\left(p^{2}+1\right)(p-1),
$$

where $p$ ranges over the primes dividing $D_{A}$.

Corollary 2 in the introduction then follows from Theorem 1 with $\mathfrak{m}=0$.

Remark 21. Theorem 13 and its Corollary 20 allow the asymptotic study of the counting of representations satisfying congruence properties. For instance, let $\Phi$ be a (nonzero) two-sided ideal in an order $O$ in a definite quaternion algebra $A$ over $\mathbb{Q}$. Let $\Gamma_{\mathscr{I}}$ be the kernel of the map $\mathrm{SL}_{2}(\mathcal{O}) \rightarrow \mathrm{GL}_{2}(\mathcal{O} / \mathscr{I})$ of reduction modulo 
$\mathscr{I}$ of the coefficients, and $\Gamma_{\mathscr{g}, 0}$ the preimage of the upper triangular subgroup by this map. Then applying Corollary 20 with $G=\Gamma_{\mathscr{I}}$ and $G=\Gamma_{\mathscr{q}, 0}$ respectively, we get an asymptotic equivalence as $s \rightarrow+\infty$ of the number of relatively prime representations $(u, v)$ of integers with absolute value at most $s$ by a given integral binary Hamiltonian form, satisfying the additional congruence properties

$$
\{u \equiv 1 \bmod \mathscr{I}, v \equiv 0 \bmod \mathscr{I}\} \quad \text { or } \quad\{v \equiv 0 \bmod \mathscr{I}\} .
$$

To give an even more precise result, the computation of the indices of $\Gamma_{\mathscr{g}}$ and $\Gamma_{\mathscr{g}, 0}$ in $\mathrm{SL}_{2}(\mathrm{O})$ would be needed.

\section{Geometric reduction theory of binary Hamiltonian forms}

Let $O$ be a (not necessarily maximal) order in a definite quaternion algebra $A$ over $\mathbb{Q}$.

Let 2 be the 6-dimensional real vector space of binary Hamiltonian forms, $2^{+}$ the open cone of positive definite ones, $2^{ \pm}$the open cone of indefinite ones, $2(0)$ the discrete subset of the ones that are integral over $\mathbb{O}$, and

$$
2^{+}(0)=2^{+} \cap 2(0), \quad 2^{ \pm}(0)=2^{ \pm} \cap 2(0) .
$$

For every $\Delta \in \mathbb{Z}-\{0\}$, let $2(\Delta)=\{f \in \mathscr{2}: \Delta(f)=\Delta\}, 2(\mathbb{0}, \Delta)=2(\Delta) \cap 2(\mathbb{0})$ and

$$
2^{+}(\mathbb{O}, \Delta)=2(\Delta) \cap \mathscr{2}^{+}(\mathbb{0}), \quad 2^{ \pm}(\mathbb{O}, \Delta)=2(\Delta) \cap \mathscr{2}^{ \pm}(\mathbb{0}) .
$$

The group $\mathbb{R}_{+}^{*}$ acts on $2^{+}$by multiplication; we will denote by $[f]$ the orbit of $f$ and by $\overline{2}^{+}$the quotient space $2^{+} / \mathbb{R}_{+}^{*}$. Similarly, the group $\mathbb{R}^{*}$ acts on $2^{ \pm}$by multiplication; we will denote by $[f]$ the orbit of $f$ and by $\overline{2}^{ \pm}$the quotient space $2^{ \pm} / \mathbb{R}^{*}$. The right action of $\mathrm{SL}_{2}(\mathbb{M})$ on 2 preserves $2(\Delta), 2^{+}$and $2^{ \pm}$, commuting with the actions of $\mathbb{R}_{+}^{*}$ and $\mathbb{R}^{*}$ on these last two spaces. The subgroup $\mathrm{SL}_{2}(\mathrm{O})$ preserves $2(0), 2^{+}(\mathbb{O}), 2^{ \pm}(0), 2^{+}(\mathbb{O}, \Delta), 2^{ \pm}(\mathbb{O}, \Delta)$.

Let $\mathscr{C}\left(\mathbb{M}_{\mathbb{R}}^{5}\right)$ be the space of totally geodesic hyperplanes of $\mathbb{M}_{\mathbb{R}}^{5}$, with the Hausdorff distance on compact subsets.

Proposition 22. (1) The map $\Phi: \overline{2}^{+} \rightarrow \mathbb{H}_{\mathbb{R}}^{5}$ defined by

$$
[f] \mapsto\left(-\frac{b(f)}{a(f)}, \frac{\sqrt{-\Delta(f)}}{a(f)}\right)
$$

is a homeomorphism, which is (anti)equivariant for the actions of $\mathrm{SL}_{2}(\mathbb{M})$ : For every $g \in \mathrm{SL}_{2}(\mathbb{W})$, we have $\Phi([f \circ g])=g^{-1} \Phi([f])$.

(2) The map $\Psi: \overline{2}^{ \pm} \rightarrow \mathscr{C}\left(\mathbb{U}_{\mathbb{R}}^{5}\right)$ defined by $[f] \mapsto \mathscr{C}(f)$ is a homeomorphism, which is (anti)equivariant for the actions of $\mathrm{SL}_{2}(\mathbb{M})$ : For every $g \in \mathrm{SL}_{2}(\mathbb{M})$, we have $\Psi([f \circ g])=g^{-1} \Psi([f])$. 
Note that $\Phi([f])$ may be geometrically understood as the pair of the center and the imaginary radius of the imaginary sphere with equation $f(z, 1)=0$, that is,

$$
\mathrm{n}\left(z+\frac{b(f)}{a(f)}\right)=-\left(\frac{\sqrt{-\Delta(f)}}{a(f)}\right)^{2} .
$$

Proof. (1) Since $a=a(f)>0$ and $\Delta=\Delta(f)<0$ when $f$ is a positive definite binary Hamiltonian form, the map $\Phi$ is well-defined and continuous. Since the orbit by $\mathbb{R}_{+}^{*}$ of a positive definite binary Hamiltonian form has a unique element $f$ such that $a(f)=1$, and since $c(f)$ then is equal to $n(b(f))-\Delta$, the map $\Phi$ is a bijection with continuous inverse $(z, r) \mapsto\left[f_{z, r}\right]$ where

$$
f_{z, r}:(u, v) \mapsto \mathrm{n}(u)-\operatorname{tr}(\bar{u} z v)+\left(\mathrm{n}(z)+r^{2}\right) \mathrm{n}(v) .
$$

To prove the equivariance property of $\Phi$, we could use (14) and the formula for the inverse of an element of $\mathrm{SL}_{2}(\mathrm{O})$ given for instance in [Kellerhals 2003], but the computations are quite technical and even longer than below. Hence we prefer to use the following lemma to decompose the computations.

Lemma 23. The group (even the monoid) $\mathrm{SL}_{2}(\mathbb{M})$ is generated by the elements

$$
\left(\begin{array}{cc}
0 & -1 \\
1 & 0
\end{array}\right) \text { and }\left(\begin{array}{ll}
1 & \beta \\
0 & 1
\end{array}\right) \quad \text { with } \beta \in \mathbb{U} \text {. }
$$

This is a consequence of a general fact about connected semisimple real Lie groups and their root groups, but the proof is short (and is one way to prove that the Dieudonné determinant of

$$
\left(\begin{array}{ll}
\alpha & \beta \\
\gamma & \delta
\end{array}\right)
$$

is $\mathrm{n}\left(\gamma \beta-\gamma \alpha \gamma^{-1} \delta\right)$ if $\left.\gamma \neq 0\right)$.

Proof. This follows from the following facts, where $\alpha, \beta, \gamma, \delta \in \mathbb{H}$. If $\alpha \neq 0$, then

$$
\begin{aligned}
\left(\begin{array}{ll}
\alpha & \beta \\
0 & \delta
\end{array}\right) & =\left(\begin{array}{ll}
\alpha & 0 \\
0 & \delta
\end{array}\right)\left(\begin{array}{cc}
1 & \alpha^{-1} \beta \\
0 & 1
\end{array}\right), \text { and } \\
\left(\begin{array}{cc}
\alpha & 0 \\
0 & \alpha^{-1}
\end{array}\right) & =\left(\begin{array}{cc}
1 & -\alpha \\
0 & 1
\end{array}\right)\left(\begin{array}{cc}
0 & -1 \\
1 & 0
\end{array}\right)\left(\begin{array}{cc}
1 & -\alpha^{-1} \\
0 & 1
\end{array}\right)\left(\begin{array}{cc}
0 & -1 \\
1 & 0
\end{array}\right)\left(\begin{array}{cc}
1 & -\alpha \\
0 & 1
\end{array}\right)\left(\begin{array}{cc}
0 & -1 \\
1 & 0
\end{array}\right) .
\end{aligned}
$$

If $\mathrm{n}(\alpha \delta)=1$, there exist $u, v \in \mathbb{W}^{\times}$such that $\alpha \delta=u v u^{-1} v^{-1}$, and

$$
\left(\begin{array}{ll}
\alpha & 0 \\
0 & \delta
\end{array}\right)=\left(\begin{array}{cc}
u & 0 \\
0 & u^{-1}
\end{array}\right)\left(\begin{array}{cc}
v & 0 \\
0 & v^{-1}
\end{array}\right)\left(\begin{array}{cc}
(v u)^{-1} & 0 \\
0 & v u
\end{array}\right)\left(\begin{array}{cc}
\delta^{-1} & 0 \\
0 & \delta
\end{array}\right) \text {. }
$$

If $\gamma \neq 0$, then

$$
\left(\begin{array}{ll}
\alpha & \beta \\
\gamma & \delta
\end{array}\right)=\left(\begin{array}{cc}
1 & \alpha \gamma^{-1} \\
0 & 1
\end{array}\right)\left(\begin{array}{cc}
0 & -1 \\
1 & 0
\end{array}\right)\left(\begin{array}{cc}
\gamma & 0 \\
0 & -\beta+\alpha \gamma^{-1} \delta
\end{array}\right)\left(\begin{array}{cc}
1 & \gamma^{-1} \delta \\
0 & 1
\end{array}\right) .
$$


Now, to prove the equivariance property, one only has to prove it for the elements of the generating set of $\mathrm{SL}_{2}(\mathbb{M})$ given in the above lemma. Given $f \in 2^{+}$, let

$$
M=\left(\begin{array}{ll}
a & b \\
\bar{b} & c
\end{array}\right)
$$

be the matrix of $f$ and $\Delta=\Delta(f)$. Note that the matrix of $f \circ g$ is $g^{*} M g$.

If

$$
g=\left(\begin{array}{ll}
1 & \beta \\
0 & 1
\end{array}\right),
$$

we have $a(f \circ g)=a$ and $b(f \circ g)=a \beta+b$. Since

$$
g^{-1} \cdot\left(-\frac{b}{a}, \frac{\sqrt{-\Delta}}{a}\right)=\left(-\frac{b}{a}-\beta, \frac{\sqrt{-\Delta}}{a}\right)=\left(-\frac{b(f \circ g)}{a(f \circ g)}, \frac{\sqrt{-\Delta(f \circ g)}}{a(f \circ g)}\right)
$$

by (16), the result follows in this case.

If

$$
g=\left(\begin{array}{cc}
0 & -1 \\
1 & 0
\end{array}\right)
$$

then $a(f \circ g)=c$ and $b(f \circ g)=-\bar{b}$. By (14), for every $(z, h) \in \mathbb{U}_{\mathbb{R}}^{5}$, we have

$$
g^{-1} \cdot(z, r)=\left(\frac{-\bar{z}}{\mathrm{n}(z)+r^{2}}, \frac{r}{\mathrm{n}(z)+r^{2}}\right) .
$$

Therefore, since $\Delta=\mathrm{n}(b)-a c$,

$$
g^{-1} \cdot\left(-\frac{b}{a}, \frac{\sqrt{-\Delta}}{a}\right)=\left(\frac{-\left(-\frac{\bar{b}}{a}\right)}{\frac{\mathrm{n}(b)}{a^{2}}+\frac{-\Delta}{a^{2}}}, \frac{\frac{\sqrt{-\Delta}}{a}}{\frac{c}{a}}\right)=\left(-\frac{b(f \circ g)}{a(f \circ g)}, \frac{\sqrt{-\Delta(f \circ g)}}{a(f \circ g)}\right) .
$$

The equivariance property of $\Phi$ follows.

(2) We have already seen that $\Psi$ is a bijection. Its equivariance property follows from (17). Let $a=a(f), b=b(f), c=c(f)$ and $\Delta=\Delta(f)$. Since

$$
\mathscr{C}(f)= \begin{cases}\left\{(z, r) \in \mathbb{U}_{\mathbb{R}}^{5}: \mathrm{n}(a z+b)+a^{2} r^{2}=\Delta\right\} & \text { if } a \neq 0, \\ \left\{(z, r) \in \mathbb{H}_{\mathbb{R}}^{5}: \operatorname{tr}(\bar{z} b)+c=0\right\} & \text { otherwise, }\end{cases}
$$

the map $\Psi$ is clearly a homeomorphism.

In order to define a geometric notion of reduced binary Hamiltonian form, much less is needed than an actual fundamental domain for the group $\mathrm{SL}_{2}(\mathrm{O})$ acting on $\mathbb{H}_{\mathbb{R}}^{5}$. Though it might increase the number of reduced elements, this will make the verification that a given binary form is reduced much easier (see the end of this section). Indeed, due to the higher dimension, the number of inequalities is much larger than the one for $\mathrm{SL}_{2}(\mathbb{Z})$ or for $\mathrm{SL}_{2}\left(O_{K}\right)$, where $\mathrm{O}_{K}$ is the ring of integers of an imaginary quadratic number field $K$; see for instance [Zagier 1981; Buchmann and Vollmer 2007; Elstrodt et al. 1998]. 
For $n \geq 2$, let us denote by $\|z\|$ the usual Euclidean norm on $\mathbb{R}^{n-1}$. Consider the upper halfspace model of the real hyperbolic $n$-space $\mathbb{U}_{\mathbb{R}}^{n}$, whose underlying manifold is $\left.\mathbb{R}^{n-1} \times\right] 0,+\infty$, so that $\partial_{\infty} \mathbb{H}_{\mathbb{R}}^{n}=\mathbb{R}^{n-1} \cup\{\infty\}$. A weak fundamental domain for the action of a finite covolume discrete subgroup $\Gamma$ of isometries of $\mathbb{U}_{\mathbb{R}}^{n}$ is a subset $\mathscr{F}$ of $\mathbb{U}_{\mathbb{R}}^{n}$ such that

(i) $\bigcup_{g \in \Gamma} g \mathscr{F}=\mathbb{U}_{\mathbb{R}}^{n}$,

(ii) there exists a compact subset $K$ in $\mathbb{R}^{n-1}$ such that $\mathscr{F}$ is contained in $\left.K \times\right] 0,+\infty[$,

(iii) there exist $\kappa, \epsilon>0$ and a finite set $Z$ of parabolic fixed points of $\Gamma$ such that $\mathscr{F}=\{(z, r) \in \mathscr{F}: r \geq \epsilon\} \cup\left(\bigcup_{s \in Z} \mathscr{E}_{s}\right)$, where $\mathscr{E}_{s} \subset\left\{(z, r) \in \mathscr{F}:\|z-s\| \leq \kappa r^{2}\right\}$.

Note that a weak fundamental domain for a finite index subgroup of $\Gamma$ is a weak fundamental domain for $\Gamma$.

When $\infty$ is a parabolic fixed point of $\Gamma$, an example of a weak fundamental domain is any Ford fundamental domain of $\Gamma$, whose definition we now recall.

Given any isometry $g$ of $\mathbb{H}_{\mathbb{R}}^{n}$ such that $g \infty \neq \infty$, the isometric sphere of $g$ is the $(n-2)$-sphere $S_{g}$ of $\mathbb{R}^{n-1}$ that consists of the points at which the tangent map of $g$ is a Euclidean isometry. We then define $S_{g}^{+}$as the set of points in $\mathbb{U}_{\mathbb{R}}^{n}$ that are in the closure of the unbounded component of the complement of the hyperbolic hyperplane whose boundary is $S_{g}$. For instance, if

$$
g=\left(\begin{array}{ll}
\alpha & \beta \\
\gamma & \delta
\end{array}\right) \in \mathrm{SL}_{2}(\mathbb{M})
$$

then $g \infty \neq \infty$ if and only if $\gamma \neq 0$ and its isometric sphere is then $S_{g}=\{z \in \mathbb{H}: \mathrm{n}(\gamma z+\delta)=1\}, \quad$ so that $\quad S_{g}^{+}=\left\{(z, r) \in \mathbb{H}_{\mathbb{R}}^{5}: \mathrm{n}(\gamma z+\delta)+r^{2} \geq 1\right\}$.

Recall that since $\Gamma$ has finite covolume, every parabolic fixed point $\xi$ of $\Gamma$ is bounded, that is, the quotient of $\partial_{\infty} \mathbb{H}_{\mathbb{R}}^{n}-\{\xi\}$ by the stabilizer of $\xi$ in $\Gamma$ is compact. Let $\mathscr{D}_{\infty}$ be a compact fundamental domain for the action of the stabilizer of $\infty$ in $\Gamma$ on $\mathbb{R}^{n-1}$. Then the Ford fundamental domain $\mathscr{F}_{\Gamma}$ of $\Gamma$ associated to $\mathscr{D}_{\infty}$ is

$$
\mathscr{F}_{\Gamma}=\left(\bigcap_{\substack{g \in \Gamma \\ g \propto \neq \infty}} S_{g}^{+}\right) \cap\left(\mathscr{D}_{\infty} \times\right] 0,+\infty[) .
$$

It is well known (see for instance [Beardon 1983, page 239]) that $\mathscr{F}_{\Gamma}$ is a fundamental domain for $\Gamma$ acting on $\mathbb{U}_{\mathbb{R}}^{n}$ (in particular, $\mathscr{F}_{\Gamma}$ satisfies condition (i) of a weak fundamental domain) and that the set of points at infinity of $\bigcap_{g \in \Gamma, g \infty \neq \infty} S_{g}^{+}$is a locally finite set of parabolic fixed points in $\partial_{\infty} \mathbb{H}_{\mathbb{R}}^{n}$. Furthermore, since parabolic fixed points are bounded and have a precisely invariant horoball centered at them, and since the tangency of a circle and its tangent is quadratic, the condition (iii) 
is satisfied for every $\epsilon$ small enough, and $\kappa$ large enough. Note that $\mathscr{F}_{\Gamma}$ satisfies condition (ii) with $K=\mathscr{D}_{\infty}$.

Let us fix a weak fundamental domain $\mathscr{F}$ for the action of $\mathrm{SL}_{2}(\mathrm{O})$ on $\mathbb{Q}_{\mathbb{R}}^{5}$. A positive definite form $f \in 2^{+}(\mathbb{O})$ is reduced if $\Phi([f]) \in \mathscr{F}$ and an indefinite form $f \in 2^{ \pm}(\mathbb{O})$ is reduced if $\Psi([f]) \cap \mathscr{F} \neq 0$. We say that a negative definite form $f \in-2^{+}(0)$ is reduced if $-f$ is reduced. The notion of being reduced does depend on the choice of a weak fundamental domain, which allows us to choose it appropriately when computing examples. Recall that $2(\Delta)$ is equal to $2^{ \pm}(\Delta)$ if $\Delta>0$ and to $2^{+}(\Delta) \cup-2^{+}(\Delta)$ if $\Delta<0$.

Theorem 24. For every $\Delta \in \mathbb{Z}-\{0\}$, the number of reduced elements of $2(\mathbb{O}, \Delta)$ is finite.

This is a restatement of Theorem 4 in the introduction.

Proof. Note that the Euclidean norm on $\mathbb{W}$ is $\|z\|=\mathrm{n}(z)^{1 / 2}$.

Let us first prove that the number of reduced elements of $2^{+}(\mathbb{O}, \Delta)$ is finite.

For every $f \in 2^{+}(\mathbb{O}, \Delta)$, let $a=a(f)>0, b=b(f)$ and $c=c(f)$. We have $\mathrm{n}(b)-a c=\Delta<0$; hence $c$ is determined by $a$ and $b$. The form $f$ is reduced if and only if

$$
\Phi([f])=\left(-\frac{b}{a}, \frac{\sqrt{-\Delta}}{a}\right) \in \mathscr{F} .
$$

By the condition (ii) and since $K$ is compact, $\|b / a\|$ is bounded. Hence, if we have an upper bound on $a$, by the discreteness of $\mathcal{O}$, the elements $a$ and $b$ may take only finitely many values, and so does $c$, therefore the result follows.

Let $\kappa, \epsilon, Z$ be as in the condition (iii). If $\sqrt{-\Delta} / a \geq \epsilon$, then $a$ is bounded from above, and we are done. Otherwise, by condition (iii), there exists $s$ in the finite set $Z$ such that $\Phi([f]) \in \mathscr{E}_{s}$. In particular,

$$
\left\|-\frac{b}{a}-s\right\| \leq \kappa\left(\frac{\sqrt{-\Delta}}{a}\right)^{2} .
$$

Since the set of parabolic elements of $\mathrm{SL}_{2}(\mathcal{O})$ is $A \cup\{\infty\}$, we may write $s=u / v$ with $u \in \mathbb{O}$ and $v \in \mathbb{N}-\{0\}$. The inequality above becomes

$$
a\|b v+a u\| \leq \kappa|\Delta| v .
$$

The element $b v+a u \in \mathbb{O}$ either is equal to 0 or has reduced norm, hence Euclidean norm, at least 1 . In the second case, we have an upper bound on $a$, as wanted. In the first case, we have $b / a=-u / v$, that is $b=-a u / v$. Hence

$$
\Delta v^{2}=(\mathrm{n}(b)-a c) v^{2}=a\left(a \mathrm{n}(u)-c v^{2}\right) .
$$

Since $a \mathrm{n}(u)-c v^{2} \in \mathbb{Z}$, the integer $a$ divides the nonzero integer $\Delta v^{2}$; hence $a$ is bounded, as wanted. 
Let us now prove that the number of reduced elements of $2^{ \pm}(0, \Delta)$ is finite, which concludes the proof of Theorem 24 .

We have $\Delta>0$. With $K$ a compact subset as in the condition (ii), let $\delta=$ $\sup _{x \in K}\|x\|$. Let $f \in \mathcal{Q}^{ \pm}(\mathcal{O}, \Delta)$ be reduced, and fix $(z, r) \in \mathscr{C}(f) \cap \mathscr{F}$. Let $a=a(f)$, $b=b(f)$ and $c=c(f)$.

Assume first that $a=0$. Then $\mathrm{n}(b)=\Delta$; hence $b$ takes only finitely many values, by the discreteness of $\mathcal{O}$. Recalling that $\mathscr{C}(f)=\left\{(z, r) \in \mathbb{U}_{\mathbb{R}}^{5}: \operatorname{tr}(\bar{z} b)+c=0\right\}$, we have by the Cauchy-Schwarz inequality

$$
|c|=|\operatorname{tr}(\bar{z} b)| \leq 2\|z\|\|b\| \leq 2 \delta \sqrt{\Delta} .
$$

Again by discreteness, $c$ takes only finitely many values, and the result follows.

Assume that $a \neq 0$, and up to replacing $f$ by $-f$ (which is reduced if $f$ is), that $a>0$. We have $\mathrm{n}(b)-a c=\Delta$, hence $c$ is determined by $a$ and $b$. Recalling that $\mathscr{b}(f)=\left\{(z, r) \in \mathbb{U}_{\mathbb{R}}^{5}: \mathrm{n}(a z+b)+a^{2} r^{2}=\Delta\right\}$, we have by the triangular inequality

$$
\left\|\frac{b}{a}\right\| \leq\left\|z+\frac{b}{a}\right\|+\|z\| \leq \sqrt{\Delta}+\delta .
$$

Hence as in the positive definite case, if we have an upper bound on $a$, the result follows.

Let $\kappa, \epsilon, Z$ be as in the condition (iii). Note that $r \leq \sqrt{\Delta} / a$. Hence if $r \geq \epsilon$, then we have an upper bound $a \leq \sqrt{\Delta} / \epsilon$, as wanted. Therefore, we may assume that $(z, r)$ belongs to $\mathscr{C}(f) \cap \mathscr{E}_{s}$ for some $s \in Z$. In particular,

$$
\left\|z+\frac{b}{a}\right\|=\sqrt{\frac{\Delta}{a^{2}}-r^{2}} \text { and }\|z-s\| \leq \kappa r^{2} .
$$

First assume that $\|(b / a)+s\| \geq \sqrt{\Delta} / a$. Then by the inverse triangular inequality

$$
\kappa r^{2} \geq\|s-z\| \geq\left\|\frac{b}{a}+s\right\|-\left\|z+\frac{b}{a}\right\| \geq \frac{\sqrt{\Delta}}{a}-\sqrt{\frac{\Delta}{a^{2}}-r^{2}} \geq \frac{r^{2}}{2 \sqrt{\Delta} / a} .
$$

Therefore, we have an upper bound $a \leq 2 \kappa \sqrt{\Delta}$, as wanted.

Now assume that $\|(b / a)+s\|<\sqrt{\Delta} / a$. Write $s=u / v$ with $u \in \mathbb{O}$ and $v \in \mathbb{N}-\{0\}$. We have $\mathrm{n}(a u+b v)<\Delta v^{2}$. The element $w=a u+b v$, belonging to 0 and having reduced norm at most $\Delta v^{2}$, can take only finitely many values. The positive integer $v^{2} \Delta-\mathrm{n}(w)$ is equal to

$$
\begin{aligned}
v^{2}(\mathrm{n}(b)-a c)-\mathrm{n}(a u+b v) & =-\operatorname{tr}(\overline{a u} b v)-\mathrm{n}(a u)-v^{2} a c \\
& =-a\left(\operatorname{tr}(\bar{u} b v)+a \mathrm{n}(u)+v^{2} c\right) .
\end{aligned}
$$

Since $\operatorname{tr}(\bar{u} b v)+a \mathrm{n}(u)+v^{2} c \in \mathbb{Z}$ by the properties of the reduced norm, the reduced trace and the conjugate of elements of $\mathbb{O}$, this implies that the integer $a$ divides the nonzero integer $v^{2} \Delta-\mathrm{n}(w)$; hence $a$ is bounded, as wanted. 
This concludes the proof of Theorem 24 .

Corollary 25. For every $\Delta \in \mathbb{Z}-\{0\}$, the number of orbits of $\mathrm{SL}_{2}(0)$ in $2(0, \Delta)$, hence in $2^{+}(0, \Delta)$ and in $2^{ \pm}(0, \Delta)$, is finite.

Proof. This immediately follows from Theorem 24, by the equivariance properties in Proposition 22 and the assumption (i) on a weak fundamental domain (that was not used in the proof of Theorem 24).

Example 26. Let $A$ be Hamilton's quaternion algebra over $\mathbb{Q}$. Let $\mathbb{O}$ be Hurwitz's maximal order in $A$, and let $O^{\prime}=\mathbb{Z}+\mathbb{Z} i+\mathbb{Z} j+\mathbb{Z} k$ be the order of Lipschitz integral quaternions.

We identify $\mathbb{H}$ and $\mathbb{R}^{4}$ by the $\mathbb{R}$-linear map sending $(1, i, j, k)$ to the canonical basis of $\mathbb{R}^{4}$. Let $V \subset O^{\prime}$ denote the set of vertices of the 4-dimensional unit cube $[0,1]^{4}$. We claim that the set

$$
\mathscr{F}=\left\{(z, r) \in \mathbb{H}_{\mathbb{R}}^{5}: z \in[0,1]^{4}, \mathrm{n}(z-s)+r^{2} \geq 1 \text { for all } s \in V\right\}
$$

is a weak fundamental domain for $\mathrm{SL}_{2}\left(\mathrm{O}^{\prime}\right)$, and hence for $\mathrm{SL}_{2}(\mathrm{O})$. For every $s \in V$, the 3 -sphere in $\mathbb{H}$ with equation $\mathrm{n}(z-s)=1$ is the isometric sphere of

$$
\left(\begin{array}{rr}
0 & -1 \\
1 & s
\end{array}\right) \in \mathrm{SL}_{2}\left(\mathbb{O}^{\prime}\right)
$$

Since the diameter of the cube $[0,1]^{4}$ is 2 , the closed balls bounded by these spheres cover $[0,1]^{4}$. This unit cube is a fundamental polytope of the subgroup of unipotent elements of $\mathrm{SL}_{2}\left(\mathrm{O}^{\prime}\right)$ fixing $\infty$. Thus, $\mathscr{F}$ contains a Ford fundamental domain of $\mathrm{SL}_{2}\left(\mathrm{O}^{\prime}\right)$, which implies property (i) of a weak fundamental domain. Property (ii) (with $K$ the unit cube) is valid by the definition of $\mathscr{F}$. Property (iii) follows from the fact that the only point at infinity of $\mathscr{F}$ besides $\infty$ is the center point $(1+i+j+k) / 2$ of the unit cube, which is the only point of this cube which does not belong to one of the open balls whose boundary is one of the isometric spheres used to define $\mathscr{F}$. Note that $(1+i+j+k) / 2 \in A$ is a parabolic fixed point of $\mathrm{SL}_{2}\left(\mathbb{O}^{\prime}\right)$.

Recall that a positive definite Hamiltonian form $f \in 2^{+}(\mathbb{O}, \Delta)$ with coefficients $a=a(f), b=b(f)=b_{1}+b_{2} i+b_{3} j+b_{4} k$ and $c=c(f)$ is reduced (for this choice of weak fundamental domain) if $(-b / a, \sqrt{-\Delta} / a) \in \mathscr{F}$. A straightforward manipulation of the defining inequalities of $\mathscr{F}$ shows that $f \in 2^{+}(\mathbb{O}, \Delta)$ is reduced if and only if its coefficients satisfy the following set of 25 inequalities

$$
a>0, \quad 0 \leq-b_{\ell} \leq a, \quad a\left(a-c-2 \sum_{m \in P} b_{m}\right) \leq \operatorname{Card}(P)
$$

for all $\ell \in\{1,2,3,4\}$ and for all subsets $P \subset\{1,2,3,4\}$. Theorem 24 implies that there are only a finite number of forms in $2^{+}(\mathcal{O}, \Delta)$ whose coefficients satisfy the inequalities (35). 
Similarly, an indefinite Hamiltonian form $f \in 2^{ \pm}(\mathbb{O}, \Delta)$ with $a(f)=a>0$, $b(f)=b_{1}+b_{2} i+b_{3} j+b_{4} k$ and $c(f)=c$ is reduced, that is, $\mathscr{C}(f)$ meets $\mathscr{F}$, if and only if the following system of 16 linear inequalities and one quadratic inequality in four real variables $X_{1}, X_{2}, X_{3}, X_{4}$ has a solution in the unit cube $[0,1]^{4}$ :

$$
\sum_{\ell=1}^{4} 2 X_{\ell} \frac{b_{\ell}}{a}+X_{\ell}^{2} \leq-\frac{c}{a}, \quad \sum_{\ell=1}^{4} 2 X_{\ell} \frac{b_{\ell}}{a}+\sum_{m \in P} 2 X_{m} \leq-1-\frac{c}{a}+\operatorname{Card}(P),
$$

for all subsets $P \subset\{1,2,3,4\}$.

\section{Appendix: The hyperbolic covolume of $\mathrm{SL}_{2}(\mathrm{O})$, by Vincent Emery}

Let $A$ be a definite quaternion algebra over $\mathbb{Q}$, with reduced discriminant $D_{A}$, and let $O$ be a maximal order in $A$; see for instance [Vignéras 1980] and Section 2 for definitions and properties. Given a quaternion algebra $A^{\prime}$ over a field $k$, let $\mathrm{SL}_{2}\left(A^{\prime}\right)=\mathrm{SL}_{1}\left(M_{2}\left(A^{\prime}\right)\right)$ be the group of elements of the central simple $2 \times 2$ matrix algebra $M_{2}\left(A^{\prime}\right)$ having reduced norm 1 . For any subring $O^{\prime}$ of $A^{\prime}$, let $\mathrm{SL}_{2}\left(\mathbb{O}^{\prime}\right)=\mathrm{SL}_{2}\left(A^{\prime}\right) \cap \mathcal{M}_{2}\left(\mathbb{O}^{\prime}\right)$ and $\mathrm{PSL}_{2}\left(\mathbb{O}^{\prime}\right)=\mathrm{SL}_{2}\left(\mathrm{O}^{\prime}\right) /\{ \pm \mathrm{id}\}$. Fixing an identification between $A \otimes_{\mathbb{Q}} \mathbb{R}$ and Hamilton's real quaternion algebra $\mathbb{U}$ turns $\mathrm{SL}_{2}(\mathrm{O})$ into an arithmetic lattice in $\mathrm{SL}_{2}(\mathbb{M})$. Hence $\mathrm{SL}_{2}(\mathrm{O})$ acts by isometries with finite covolume on the real hyperbolic space $\mathbb{T}_{\mathbb{R}}^{5}$; see for instance Section 3 for generalities.

In this appendix, the following result is proved using Prasad's volume formula in [Prasad 1989]. See the main body of this paper for a proof using Eisenstein series.

Theorem A.1. The hyperbolic covolume of $\mathrm{SL}_{2}(\mathrm{O})$ is

$$
\operatorname{Covol}\left(\mathrm{SL}_{2}(\mathcal{O})\right)=\frac{\zeta(3)}{11520} \prod_{p \mid D_{A}}\left(p^{3}-1\right)(p-1)
$$

where $p$ ranges over the prime integers.

Proof. Let $\mathscr{P}$ be the set of positive primes in $\mathbb{Z}$. For every $p \in \mathscr{P}$, let $\mathcal{O}_{p}=0 \otimes_{\mathbb{Z}} \mathbb{Z}_{p}$, which is a maximal order in the quaternion algebra $A_{p}=A \otimes_{\mathbb{Q}} \mathbb{Q}_{p}$ over $\mathbb{Q}_{p}$; see for instance [Vignéras 1980, page 84].

We refer for instance to [Tits 1966] for the classification of the semisimple connected algebraic groups over $\mathbb{Q}$. Let $\mathbf{G}$ be the (affine) algebraic group over $\mathbb{Q}$, having as its group of $K$-points, for each characteristic zero field $K$, the group

$$
\mathbf{G}(K)=\mathrm{SL}_{2}\left(A \otimes_{\mathbb{Q}} K\right)=\mathrm{SL}_{1}\left(M_{2}\left(A \otimes_{\mathbb{Q}} K\right)\right) .
$$

The group $\mathbf{G}$ is absolutely (quasi)simple and simply connected. Indeed, the $\mathbb{C}$ algebra $A \otimes_{\mathbb{Q}} \mathbb{C}$ is isomorphic to $\mu_{2}(\mathbb{C})$ and thus the complex Lie group $\mathbf{G}(\mathbb{C})$ is isomorphic to $\mathrm{SL}_{1}\left(M_{4}(\mathbb{C})\right)=\mathrm{SL}_{4}(\mathbb{C})$ (note that we are using the reduced norm 
and not the norm). Furthermore, $\mathbf{G}$ is an inner form of the split algebraic group $\mathscr{G}=\mathrm{SL}_{4}$ over $\mathbb{Q}$. The (absolute) rank of $\mathscr{G}$ and the exponents of $\mathscr{G}$ are given by

$$
r=3 \quad \text { and } \quad m_{1}=1, m_{2}=2, m_{3}=3
$$

see for instance [Prasad 1989, page 96]. We consider the $\mathbb{Z}$-form of $\mathbf{G}$ such that $\mathbf{G}(\mathbb{Z})=\mathrm{SL}_{2}(\mathcal{O})$ and $\mathbf{G}\left(\mathbb{Z}_{p}\right)=\mathrm{SL}_{2}\left(\mathscr{O}_{p}\right)$ for every $p \in \mathscr{P}$; see for instance [Parkkonen and Paulin 2010, page 382] for details.

Let $\mathscr{I}_{\mathbf{G}, \mathbb{Q}_{p}}$ be the Bruhat-Tits building of $\mathbf{G}$ over $\mathbb{Q}_{p}$; see for instance [Tits 1979] for the necessary background on Bruhat-Tits theory. Recall that a subgroup of $\mathbf{G}\left(\mathbb{Q}_{p}\right)$ is parahoric if it is the stabilizer of a simplex of $\Phi_{\mathbf{G}, \mathbb{Q}_{p}}$; a coherent family of parahoric subgroups of $\mathbf{G}$ is a family $\left(Y_{p}\right)_{p \in \mathscr{P}}$, where $Y_{p}$ is a parahoric subgroup of $\mathbf{G}\left(\mathbb{Q}_{p}\right)$ and $Y_{p}=\mathbf{G}\left(\mathbb{Z}_{p}\right)$ for $p$ big enough. The principal lattice associated with this family is the subgroup $\mathbf{G}(\mathbb{Q}) \cap \prod_{p} Y_{p}$ of $\mathbf{G}(\mathbb{Q})$ (diagonally contained in the group $\mathbf{G}\left(\mathbb{A}_{f}\right)=\prod_{p}^{\prime} \mathbf{G}\left(\mathbb{Q}_{p}\right)$ of finite adèles of $\mathbf{G}$, where as usual $\prod^{\prime}$ indicates the restricted product).

For every $p \in \mathscr{P}$, recall that by the definition of the discriminant $D_{A}$ of $A$, if $p$ does not divide $D_{A}$, then the algebra $A_{p}$ is isomorphic to $M_{2}\left(\mathbb{Q}_{p}\right)$, and otherwise $A_{p}$ is a $d^{2}$-dimensional central division algebra with center $\mathbb{Q}_{p}$ with $d=2$. Furthermore, for the discrete valuation $v=v_{p} \circ \mathrm{n}$, where $v_{p}$ is the discrete valuation of $\mathbb{Q}_{p}$ and $\mathrm{n}$ the reduced norm on $A_{p}$, the maximal order $\mathrm{O}_{p}$ is equal to the valuation ring of $v$; see for instance [Vignéras 1980, page 34].

First assume that $p$ does not divide $D_{A}$. Then $\mathbf{G}$ is isomorphic to $\mathscr{G}=\mathrm{SL}_{4}$ over $\mathbb{Q}_{p}$. The vertices of the building $\mathscr{I}_{\mathbf{G}, \mathbb{Q}_{p}}$ are the homothety classes of $\mathbb{Z}_{p}$-lattices in $\mathbb{Q}_{p}{ }^{4}$. In particular $\mathrm{SL}_{2}\left(O_{p}\right)=\mathrm{SL}_{4}\left(\mathbb{Z}_{p}\right)$ is the stabilizer of the class of the standard $\mathbb{Z}_{p}$-lattice $\mathbb{Z}_{p}{ }^{4}$ and hence is parahoric.

Now assume that $p$ divides $D_{A}$. Then $\mathbf{G}\left(\mathbb{Q}_{p}\right)=\operatorname{SL}_{m}\left(A_{p}\right)$ with $m=2$ and $\mathbf{G}\left(\mathbb{Q}_{p}\right)$ has local type ${ }^{d} A_{m d-1}={ }^{2} A_{3}$ in Tits' classification [1979, Section 4.4]. The corresponding local index is shown below:

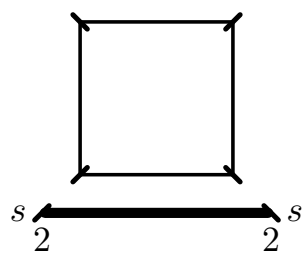

Local index of type ${ }^{2} A_{3}$.

The building $\mathscr{I}_{\mathbf{G}, \mathbb{Q}_{p}}$ is a tree (see for instance [Serre 1977] for the construction of the Bruhat-Tits tree of $\mathrm{SL}_{2}(K)$ even when $K$ is a noncommutative division algebra endowed with a discrete valuation). Its vertices are the homothety classes of 
$\mathrm{O}_{p}$-lattices in the right $A_{p}$-vector space $A_{p}{ }^{2}$. In particular $\mathrm{SL}_{2}\left(\mathrm{O}_{p}\right)$ is the stabilizer of the class of the standard $\mathrm{O}_{p}$-lattice $\mathrm{O}_{p}{ }^{2}$, hence is parahoric.

Therefore, by definition, the family $\left(\mathrm{SL}_{2}\left(\mathcal{O}_{p}\right)\right)_{p \in \mathscr{P}}$ is a coherent family of (maximal) parahoric subgroups of $\mathbf{G}$, and $\mathrm{SL}_{2}(\mathcal{O})=\mathbf{G}(\mathbb{Z})=\mathbf{G}(\mathbb{Q}) \cap \prod_{p \in \mathscr{P}} \mathbf{G}\left(\mathbb{Z}_{p}\right)$ is its associated principal lattice.

For every $p \in \mathscr{P}$, let $\bar{M}_{p}$ (respectively $\bar{M}_{p}$ ) be the maximal reductive quotient, defined over the residual field $\mathbb{F}_{p}=\mathbb{Z}_{p} / p \mathbb{Z}_{p}$, of the identity component of the reduction modulo $p$ of the smooth affine group scheme over $\mathbb{Z}_{p}$ associated with the vertex of $\mathscr{I}_{\mathbf{G}, \mathbb{Q}_{p}}$ (respectively $\mathscr{I}_{\mathscr{G}_{,} \mathbb{Q}_{p}}$ ) stabilized by the parahoric subgroup $\mathrm{SL}_{2}\left(O_{p}\right)$ (respectively $\mathrm{SL}_{4}\left(\mathbb{Z}_{p}\right)$ ); see for instance [Tits 1979, Section 3.5]. Note that $\bar{M}_{p}=\bar{M}_{p}$ if $p$ does not divide $D_{A}$, and that for every $p \in \mathscr{P}$ the algebraic group $\bar{M}_{p}$ is isomorphic to $\mathrm{SL}_{4}$ over $\mathbb{F}_{p}$. In particular $\bar{M}_{p}\left(\mathbb{F}_{p}\right)=\mathrm{SL}_{4}\left(\mathbb{F}_{p}\right)$ and thus, for every $p \in \mathscr{P}$, the orders of finite groups of Lie type being listed for example in [Ono 1966, Table 1], we have

$$
\operatorname{dim} \bar{M}_{p}=15 \text { and }\left|\overline{\mathcal{M}}_{p}\left(\mathbb{F}_{p}\right)\right|=p^{6}\left(p^{2}-1\right)\left(p^{3}-1\right)\left(p^{4}-1\right) .
$$

If $p$ divides $D_{A}$, by applying the theory in [Tits 1979, §3.5.2] on the local index ${ }^{2} A_{3}$, we see that the semisimple part $\bar{M}_{p}^{s s}$ of $\bar{M}_{p}$ (given as the commutator algebraic group $\left.\left[\bar{M}_{p}, \bar{M}_{p}\right]\right)$ is of type ${ }^{2}\left(A_{1} \times A_{1}\right)$ and the radical $R\left(\bar{M}_{p}\right)$ of $\bar{M}_{p}$ must be a one-dimensional nonsplit torus over $\mathbb{F}_{p}$. In particular $\left|R\left(\bar{M}_{p}\right)\left(\mathbb{F}_{p}\right)\right|=p+1$ and $\bar{M}_{p}^{s s}\left(\mathbb{F}_{p}\right)$ has the same order as $\mathrm{SL}_{2}\left(\mathbb{F}_{p^{2}}\right)$, that is, $p^{2}\left(p^{4}-1\right)$. Since the radical $R\left(\bar{M}_{p}\right)$ is central in $\bar{M}_{p}$ and the intersection $R\left(\bar{M}_{p}\right) \cap \bar{M}_{p}^{s s}$ is finite (see [Springer 1998, Proposition 7.3.1]), the product map

$$
\bar{M}_{p}^{s s} \times R\left(\bar{M}_{p}\right) \rightarrow \bar{M}_{p}, \quad(x, y) \mapsto x y
$$

is an isogeny (defined over $\mathbb{F}_{p}$ ) and using Lang's isogeny theorem (see for example [Platonov and Rapinchuk 1994, Proposition 6.3, page 290]), we obtain the order of $\bar{M}_{p}\left(\mathbb{F}_{p}\right)$ as the product $\left|\bar{M}_{p}^{s s}\left(\mathbb{F}_{p}\right)\right| \cdot\left|R\left(\bar{M}_{p}\right)\left(\mathbb{F}_{p}\right)\right|$.

Alternatively, the order of $\bar{M}_{p}\left(\mathbb{F}_{p}\right)$ can be deduced from the concrete structure of $\bar{M}_{p}$ given in [Bruhat and Tits 1984]. Namely, it follows from [ibid., Proposition 3.11 and Section 5.5] that $\bar{M}_{p}\left(\mathbb{F}_{p}\right)$ corresponds to the group of elements of reduced norm 1 in the $\mathbb{F}_{p}$-algebra $M_{2}\left(\mathbb{F}_{p^{2}}\right)$ (where $\mathbb{F}_{p^{2}}$ appears as the residue field of the division algebra $A_{p}$; see [Vignéras 1980, page 35]). The reduced norm (over $\mathbb{F}_{p}$ ) of an element $g \in M_{2}\left(\mathbb{F}_{p^{2}}\right)$ is $N_{\mathbb{F}_{p}^{2} \mid \mathbb{F}_{p}}(\operatorname{det}(g))$, where $N_{\mathbb{F}_{p^{2}} \mid \mathbb{F}_{p}}$ is the norm of the extension $\mathbb{F}_{p^{2}} \mid \mathbb{F}_{p}$. Thus $\bar{M}_{p}\left(\mathbb{F}_{p}\right)$ is the kernel of the surjective homomorphism $\mathrm{GL}_{2}\left(\mathbb{F}_{p^{2}}\right) \rightarrow \mathbb{F}_{p}^{\times}$defined by $g \mapsto \operatorname{det}(g)^{p+1}$.

Therefore, from any of the two arguments above, we obtain that for every $p \in \mathscr{P}$ dividing $D_{A}$,

$$
\operatorname{dim} \bar{M}_{p}=7 \quad \text { and } \quad\left|\bar{M}_{p}\left(\mathbb{F}_{p}\right)\right|=p^{2}\left(p^{4}-1\right)(p+1) .
$$


Let $\mu$ be the Haar measure on $\mathbf{G}(\mathbb{R})=\mathrm{SL}_{2}(\mathbb{M})$ normalized as in [Prasad 1989]. That is, if $w$ is the top degree exterior form on the real Lie algebra of $\mathbf{G}(\mathbb{R})$ whose associated invariant differential form on $\mathbf{G}(\mathbb{R})$ defines the measure $\mu$ and if $\mathbf{G}_{u}(\mathbb{R})$ is a compact real form of $\mathbf{G}(\mathbb{C})$, then the complexification $w_{\mathbb{C}}$ of $w$ on the complex Lie algebra of $\mathbf{G}(\mathbb{C})=\mathbf{G}_{u}(\mathbb{C})$ defines a top degree exterior form $w_{u}$ on the real Lie algebra of $\mathbf{G}_{u}(\mathbb{R})$, whose associated invariant differential form on $\mathbf{G}_{u}(\mathbb{R})$ defines a measure $\mu_{u}$, and we require that $\mu_{u}\left(\mathbf{G}_{u}(\mathbb{R})\right)=1$.

Let $\mu^{\prime}$ be the Haar measure on $\mathrm{PSL}_{2}(\mathbb{M})=\mathrm{SO}_{0}(1,5)$ that disintegrates by the fibration $\mathrm{SO}_{0}(1,5) \rightarrow \mathrm{SO}_{0}(1,5) / \mathrm{SO}(5)=\mathbb{U}_{\mathbb{R}}^{5}$ with measures on the fibers of total mass one 1 and measure on the base the Riemannian measure $d \operatorname{vol}_{\mathbb{\mathbb { N } _ { R } ^ { 5 }}}$ of the Riemannian metric of constant sectional curvature -1 . Let $\tilde{\mu}^{\prime}$ be the Haar measure on $\mathrm{SL}_{2}(\mathbb{M})$ such that the tangent map at the identity of the double cover of real Lie groups $\mathrm{SL}_{2}(\mathbb{M}) \rightarrow \mathrm{PSL}_{2}(\mathbb{R})$ preserves the top degree exterior forms defining the Haar measures. In particular, since - id belongs to $\mathrm{SL}_{2}(\mathrm{O})$,

$$
\begin{aligned}
\operatorname{Covol}\left(\mathrm{SL}_{2}(\mathbb{O})\right) & =\operatorname{Vol}\left(\mathrm{PSL}_{2}(\mathbb{O}) \backslash \mathbb{T}_{\mathbb{R}}^{5}\right) \\
& =\mu^{\prime}\left(\mathrm{PSL}_{2}(\mathbb{O}) \backslash \mathrm{PSL}_{2}(\mathbb{M})\right)=\tilde{\mu}^{\prime}\left(\mathrm{SL}_{2}(\mathcal{O}) \backslash \mathrm{SL}_{2}(\mathbb{M})\right) .
\end{aligned}
$$

Similarly, with $\mathbb{S}_{5}$ the 5-sphere endowed with its standard Riemannian metric of constant sectional curvature +1 , let $\mu_{u}^{\prime}$ be the Haar measure on $\mathrm{SO}(6)$ that disintegrates by the fibration $\mathrm{SO}(6) \rightarrow \mathrm{SO}(6) / \mathrm{SO}(5)=\mathbb{S}_{5}$ with measures on the fibers of total mass one 1 and measure on the base the Riemannian measure. In particular, $\mu_{u}^{\prime}(\mathrm{SO}(6))=\operatorname{Vol}\left(\mathbb{S}_{5}\right)$. Recall that

$$
\operatorname{Vol}\left(\mathbb{S}_{n}\right)=\frac{2 \pi^{m}}{(m-1) !} \quad \text { if } n=2 m-1 \geq 3
$$

It is well known (see for instance [Helgason 1978]) that the duality $G / K \mapsto G_{u} / K$ between irreducible symmetric spaces of noncompact type endowed with a left invariant Riemannian metric and the ones of compact type, where $G_{u}$ is a compact form of the complexification of $G$, sends $\mathbb{H}_{\mathbb{R}}^{5}$ to $\mathbb{S}_{5}$, and hence $\mu^{\prime}$ to $\mu_{u}^{\prime}$.

The maximal compact subgroup SU(4) of $\mathrm{SL}_{4}(\mathbb{C})$ is a covering of degree 2 of $\mathrm{SO}(6)$, which is the compact real form corresponding to $\mathrm{SO}_{0}(1,5)$. Hence we have (as first proved in [Emery 2009, Section 13.3])

$$
\tilde{\mu}^{\prime}=2 \operatorname{Vol}\left(\mathbb{S}_{5}\right) \mu=2 \pi^{3} \mu .
$$

By Prasad's volume formula [Prasad 1989, Theorem 3.7] (where with the notation of this theorem, $\ell=k=\mathbb{Q}$ (hence $D_{k}=D_{\ell}=1$ ), $S=V_{\infty}=\{\infty\}$ and the Tamagawa number $\tau_{\mathbb{Q}}(\mathbf{G})$ is 1 ), we have, since $\bar{M}_{p}=\bar{M}_{p}$ if $p$ does not divide $D_{A}$ and by (A1) 
for the second equality,

$$
\begin{gathered}
\mu\left(\mathrm{SL}_{2}(\mathbb{O}) \backslash \mathrm{SL}_{2}(\mathbb{M})\right)=\prod_{i=1}^{r} \frac{\left(m_{i}\right) !}{(2 \pi)^{m_{i}+1}} \prod_{p \in \mathscr{P}} \frac{p^{\left(\operatorname{dim} \bar{M}_{p}+\operatorname{dim} \bar{M}_{p}\right) / 2}}{\left|\bar{M}_{p}\left(\mathbb{F}_{p}\right)\right|} \\
=\frac{12}{(2 \pi)^{9}} \prod_{p \in \mathscr{P}} \frac{p^{\operatorname{dim} \bar{M}_{p}}}{\left|\bar{M}_{p}\left(\mathbb{F}_{p}\right)\right|} \prod_{p \mid D_{A}} \frac{\left|\bar{M}_{p}\left(\mathbb{F}_{p}\right)\right|}{\left|\bar{M}_{p}\left(\mathbb{F}_{p}\right)\right|} p^{\left(\operatorname{dim} \bar{M}_{p}-\operatorname{dim} \overline{\mathcal{M}}_{p}\right) / 2} .
\end{gathered}
$$

Using Euler's product formula $\zeta(s)=\prod_{p \in \mathscr{P}} 1 /\left(1-p^{-s}\right)$ for Riemann's zeta function, we have by (A2), since $\zeta(2)=\pi^{2} / 6$ and $\zeta(4)=\pi^{4} / 90$,

$$
\prod_{p \in \mathscr{P}} \frac{p^{\operatorname{dim} \overline{\mathcal{M}}_{p}}}{\left|\overline{\mathcal{M}}_{p}\left(\mathbb{F}_{p}\right)\right|}=\zeta(2) \zeta(3) \zeta(4)=\frac{\pi^{6} \zeta(3)}{540} \text {. }
$$

Using the equations (A4), (A5), (A6), (A7), (A2) and (A3), the result follows.

\section{Acknowledgments}

J. Parkkonen and F. Paulin thank P. Sarnak for his comments on the origin of volume computations using Eisenstein series, G. Chenevier for the proof of Lemma 6, Y. Benoist and F. Choucroun for discussions related to the appendix, and the referee for helpful comments, in particular for Lemma 23. F. Paulin thanks the University of Jyväskylä for the nice snow and its financial support. V. Emery thanks J. Parkkonen and F. Paulin for helpful discussions. He is particularly grateful to F. Paulin for his help concerning the Bruhat-Tits buildings appearing in the proof of Theorem A.1.

\section{References}

[Aslaksen 1996] H. Aslaksen, “Quaternionic determinants”, Math. Intelligencer 18 (1996), 57-65. MR 97j:16028 Zbl 0881.15007

[Beardon 1983] A. F. Beardon, The geometry of discrete groups, Grad. Texts in Math. 91, Springer, 1983. MR 85d:22026 Zbl 0528.30001

[Borel 1966] A. Borel, "Reduction theory for arithmetic groups", pp. 20-25 in Algebraic groups and discontinuous subgroups (Boulder, 1965), edited by A. Borel and G. D. Mostow, Proc. Sympos. Pure Math. IX, American Mathematical Society, 1966. MR 34 \#4372 Zbl 0213.47201

[Borel and Harish-Chandra 1962] A. Borel and Harish-Chandra, "Arithmetic subgroups of algebraic groups", Ann. of Math. 75 (1962), 485-535. MR 26\#5081 Zbl 0107.14804

[Bourbaki 1959] N. Bourbaki, Algèbre Chapitre 9: Formes sesquilinéaires et formes quadratiques, Hermann, 1959. MR 0107661 Zbl 0102.25503

[Breulmann and Helmke 1996] S. Breulmann and V. Helmke, "The covolume of quaternion groups on the four-dimensional hyperbolic space”, Acta Arith. 77 (1996), 9-21. MR 97i:11051 Zbl 0848.11018 [Bruhat and Tits 1972] F. Bruhat and J. Tits, "Groupes réductifs sur un corps local”, Publ. Math. Inst. Hautes Études Sci. 41 (1972), 5-251. MR 48 \#6265 Zbl 0254.14017 
[Bruhat and Tits 1984] F. Bruhat and J. Tits, "Schémas en groupes et immeubles des groupes classiques sur un corps local”, Bull. Soc. Math. France 112 (1984), 259-301. MR 86i:20064 Zbl 0565.14028

[Buchmann and Vollmer 2007] J. Buchmann and U. Vollmer, Binary quadratic forms: An algorithmic approach, Algorithms Comput. Math. 20, Springer, 2007. MR 2008b:11046 Zbl 1125.11028

[Cassels 1978] J. W. S. Cassels, Rational quadratic forms, London Math. Soc. Monogr. 13, Academic Press, 1978. Zbl 0395.10029

[Deuring 1968] M. Deuring, Algebren, 2nd ed., Ergeb. Math. Grenzgeb. 41, Springer, 1968. MR 37 \#4106 Zbl 0159.04201

[Dieudonné 1943] J. Dieudonné, "Les déterminants sur un corps non commutatif”, Bull. Soc. Math. France 71 (1943), 27-45. MR 7,3a Zbl 0028.33904

[Eichler 1938] M. Eichler, “Über die Idealklassenzahl total definiter Quaternionenalgebren”, Math. Z. 43 (1938), 102-109. MR 1545717 JFM 3.0093.02

[Elstrodt et al. 1998] J. Elstrodt, F. Grunewald, and J. Mennicke, Groups acting on hyperbolic space: Harmonic analysis and number theory, Springer, 1998. MR 98g:11058 Zbl 0888.11001

[Emery 2009] V. Emery, Du volume des quotients arithmétiques de l'espace hyperbolique, thèse $n^{\mathrm{o}}$ 1648, Université de Fribourg (Suisse), 2009, available at http://www.unige.ch/math/folks/emery/ Emery.pdf.

[Eskin et al. 1991] A. Eskin, Z. Rudnick, and P. Sarnak, "A proof of Siegel's weight formula", Internat. Math. Res. Notices 1991:5 (1991), 65-69. MR 92m:11040 Zbl 0743.11023

[Hashimoto and Ibukiyama 1980] K.-i. Hashimoto and T. Ibukiyama, "On class numbers of positive definite binary quaternion Hermitian forms, I”, J. Fac. Sci. Univ. Tokyo Sect. IA Math. 27 (1980), 549-601. MR 82j:10038 Zbl 0452.10029

[Hashimoto and Ibukiyama 1981] K.-i. Hashimoto and T. Ibukiyama, "On class numbers of positive definite binary quaternion Hermitian forms, II”, J. Fac. Sci. Univ. Tokyo Sect. IA Math. 28 (1981), 695-699. MR 83m:10029 Zbl 0493.10030

[Hashimoto and Ibukiyama 1983] K.-i. Hashimoto and T. Ibukiyama, "On class numbers of positive definite binary quaternion Hermitian forms, III”, J. Fac. Sci. Univ. Tokyo Sect. IA Math. 30 (1983), 393-401. MR 85i:11030 Zbl 0533.10019

[Helgason 1978] S. Helgason, Differential geometry, Lie groups, and symmetric spaces, Pure and Applied Math. 80, Academic Press, 1978. MR 80k:53081 Zbl 0451.53038

[Hild 2007] T. Hild, "The cusped hyperbolic orbifolds of minimal volume in dimensions less than ten”, J. Algebra 313 (2007), 208-222. MR 2008g:57038 Zbl 1119.52011

[Johnson and Weiss 1999] N. W. Johnson and A. I. Weiss, "Quaternionic modular groups", Linear Algebra Appl. 295 (1999), 159-189. MR 2000j:20096 Zbl 0960.20031

[Kellerhals 2003] R. Kellerhals, "Quaternions and some global properties of hyperbolic 5-manifolds", Canad. J. Math. 55 (2003), 1080-1099. MR 2005b:57032 Zbl 1054.57019

[Krafft and Osenberg 1990] V. Krafft and D. Osenberg, "Eisensteinreihen für einige arithmetisch definierte Untergruppen von $\mathrm{SL}_{2}(\mathbf{H})$ ”, Math. Zeit. 204 (1990), 425-449. MR 92f:11067 Zbl 0725. 11024

[Langlands 1966] R. P. Langlands, "The volume of the fundamental domain for some arithmetical subgroups of Chevalley groups", pp. 143-148 in Algebraic groups and discontinuous subgroups (Boulder, 1965), edited by A. Borel and G. D. Mostow, Proc. Sympos. Pure Math. IX, American Math. Soc., 1966. MR 35 \#4226 Zbl 0218.20041 
[Magnus et al. 1966] W. Magnus, F. Oberhettinger, and R. P. Soni, Formulas and theorems for the special functions of mathematical physics, 3rd ed., Grund. math. Wiss. 52, Springer, 1966. MR 38 \#1291 Zbl 0143.08502

[Ono 1966] T. Ono, "On algebraic groups and discontinuous groups", Nagoya Math. J. 27 (1966), 279-322. MR 33 \#7342 Zbl 0166.29802

[Parkkonen and Paulin 2010] J. Parkkonen and F. Paulin, "Prescribing the behaviour of geodesics in negative curvature”, Geom. Topol. 14 (2010), 277-392. MR 2011a:53060 Zbl 1191.53026

[Parkkonen and Paulin 2011] J. Parkkonen and F. Paulin, "On the representation of integers by indefinite binary Hermitian forms", Bull. Lond. Math. Soc. 43 (2011), 1048-1058. MR 2861527 Zbl 05989570

[Parkkonen and Paulin 2012] J. Parkkonen and F. Paulin, "Équidistribution, comptage et approximation par irrationnels quadratiques", J. Mod. Dyn. 6 (2012), 1-40. MR 2929128 Zbl 06049546

[Platonov and Rapinchuk 1994] V. Platonov and A. Rapinchuk, Algebraic groups and number theory, Pure and Applied Mathematics 139, Academic Press, 1994. MR 95b:11039 Zbl 0841.20046

[Prasad 1989] G. Prasad, "Volumes of S-arithmetic quotients of semi-simple groups", Publ. Math. Inst. Hautes Études Sci. 69 (1989), 91-117. MR 91c:22023 Zbl 0695.22005

[Pronin 1967] L. N. Pronin, "Integral binary Hermitian forms over the skewfield of quaternions", Vestnik Har'kov. Gos. Univ. 1967:26 (1967), 27-41. In Russian. MR 40 \#2606 Zbl 0258.10008

[Rankin 1939a] R. A. Rankin, "Contributions to the theory of Ramanujan's function $\tau(n)$ and similar arithmetical functions, I: The zeros of the function $\sum_{n=1}^{\infty} \tau(n) / n^{s}$ on the line $\Re s=13 / 2$ ", Proc. Cambridge Philos. Soc. 35 (1939), 351-356. MR 1,69d Zbl 0021.39201

[Rankin 1939b] R. A. Rankin, "Contributions to the theory of Ramanujan's function $\tau(n)$ and similar arithmetical functions, II: The order of the Fourier coefficients of integral modular forms", Proc. Cambridge Philos. Soc. 35 (1939), 357-372. MR 1,69d Zbl 0021.39202

[Reiner 1975] I. Reiner, Maximal orders, London Math. Soc. Monogr. 5, Academic Press, 1975. MR 52 \#13910 Zbl 0305.16001

[Sarnak 1983] P. Sarnak, "The arithmetic and geometry of some hyperbolic three-manifolds", Acta Math. 151 (1983), 253-295. MR 85d:11061

[Schoeneberg 1939] B. Schoeneberg, "Über die $\zeta$-Funktion einfacher hyperkomplexer Systeme", Math. Ann. 117 (1939), 85-88. MR 1,203e Zbl 0021.38802

[Selberg 1940] A. Selberg, "Bemerkungen über eine Dirichletsche Reihe, die mit der Theorie der Modulformen nahe verbunden ist", Arch. Math. Naturvid. 43 (1940), 47-50. MR 2,88a JFM 66.0377.01

[Serre 1977] J.-P. Serre, Arbres, amalgames, $\mathrm{SL}_{2}$, Astérisque 46, Soc. Math. de France, 1977. MR 57 \#16426 Zbl 0369.20013

[Springer 1998] T. A. Springer, Linear algebraic groups, 2nd ed., Prog. Math. 9, Birkhäuser, Boston, MA, 1998. MR 99h:20075 Zbl 0927.20024

[Tits 1966] J. Tits, "Classification of algebraic semisimple groups", pp. 33-62 in Algebraic groups and discontinuous subgroups (Boulder, 1965), edited by A. Borel and G. D. Mostow, Proc. Sympos. Pure Math. IX, American Mathematical Society, 1966. MR 37 \#309 Zbl 0238.20052

[Tits 1979] J. Tits, "Reductive groups over local fields", pp. 29-69 in Automorphic forms, representations and L-functions, I (Corvallis, 1977), edited by A. Borel and W. Casselman, Proc. Sympos. Pure Math. XXXIII, American Mathematical Society, 1979. MR 80h:20064

[Vignéras 1980] M.-F. Vignéras, Arithmétique des algèbres de quaternions, Lecture Notes in Mathematics 800, Springer, 1980. MR 82i:12016 Zbl 0422.12008 
[Weyl 1940] H. Weyl, "Theory of reduction for arithmetical equivalence, I", Trans. Amer. Math. Soc. 48 (1940), 126-164. MR 2,35h Zbl 0024.14802

[Weyl 1942] H. Weyl, "Theory of reduction for arithmetical equivalence, II", Trans. Amer. Math. Soc. 51 (1942), 203-231. MR 2,35h Zbl 0028.01201

[Zagier 1981] D. B. Zagier, Zetafunktionen und quadratische Körper: Eine Einführung in die höhere Zahlentheorie, Springer, 1981. MR 82m:10002 Zbl 0459.10001

Communicated by Marie-France Vignéras

Received 2011-05-11 Revised 2011-12-14 Accepted 2012-01-30

parkkone@maths.jyu.fi Department of Mathematics and Statistics, University of Jyväskylä, P.O. Box 35 , Fl-40014 University of Jyväskylä, Finland

frederic.paulin@math.u-psud.fr Département de mathématique, UMR 8628 CNRS, Université Paris-Sud, Bât. 425, 91405 ORSAY Cedex, France vincent.emery@gmail.com Section de mathématiques, 2-4 rue du Lièvre, Case postale 64, 1211 Genève 4, Switzerland 


\section{Algebra \& Number Theory}

msp.org/ant

\section{EDITORS}

MANAGING EDITOR

Bjorn Poonen

Massachusetts Institute of Technology

Cambridge, USA

\author{
EDITORIAL BOARD CHAIR \\ David Eisenbud \\ University of California \\ Berkeley, USA
}

\section{BOARD OF EDITORS}

Georgia Benkart

Dave Benson

Richard E. Borcherds

John H. Coates

J-L. Colliot-Thélène

Brian D. Conrad

Hélène Esnault

Hubert Flenner

Edward Frenkel

Andrew Granville

Joseph Gubeladze

Ehud Hrushovski

Craig Huneke

Mikhail Kapranov

Yujiro Kawamata

János Kollár

Yuri Manin

Barry Mazur

Philippe Michel
University of Wisconsin, Madison, USA

University of Aberdeen, Scotland

University of California, Berkeley, USA

University of Cambridge, UK

CNRS, Université Paris-Sud, France

University of Michigan, USA

Freie Universität Berlin, Germany

Ruhr-Universität, Germany

University of California, Berkeley, USA

Université de Montréal, Canada

San Francisco State University, USA

Hebrew University, Israel

University of Virginia, USA

Yale University, USA

University of Tokyo, Japan

Princeton University, USA

Northwestern University, USA

Harvard University, USA

École Polytechnique Fédérale de Lausanne
Susan Montgomery

Shigefumi Mori

Raman Parimala

Jonathan Pila

Victor Reiner

Karl Rubin

Peter Sarnak

Joseph H. Silverman

Michael Singer

Vasudevan Srinivas

J. Toby Stafford

Bernd Sturmfels

Richard Taylor

Ravi Vakil

Michel van den Bergh

Marie-France Vignéras

Kei-Ichi Watanabe

Andrei Zelevinsky

Efim Zelmanov
University of Southern California, USA

RIMS, Kyoto University, Japan

Emory University, USA

University of Oxford, UK

University of Minnesota, USA

University of California, Irvine, USA

Princeton University, USA

Brown University, USA

North Carolina State University, USA

Tata Inst. of Fund. Research, India

University of Michigan, USA

University of California, Berkeley, USA

Harvard University, USA

Stanford University, USA

Hasselt University, Belgium

Université Paris VII, France

Nihon University, Japan

Northeastern University, USA

University of California, San Diego, USA

\section{PRODUCTION}

production@msp.org

Silvio Levy, Scientific Editor

See inside back cover or msp.org/ant for submission instructions.

The subscription price for 2013 is US \$200/year for the electronic version, and \$350/year ( $\$ 40$, if shipping outside the US) for print and electronic. Subscriptions, requests for back issues and changes of subscribers address should be sent to MSP.

Algebra \& Number Theory (ISSN 1944-7833 electronic, 1937-0652 printed) at Mathematical Sciences Publishers, 798 Evans Hall \#3840, c/o University of California, Berkeley, CA 94720-3840 is published continuously online. Periodical rate postage paid at Berkeley, CA 94704, and additional mailing offices.

ANT peer review and production are managed by EditFLOW ${ }^{\circledR}$ from Mathematical Sciences Publishers.

\section{PUBLISHED BY}

mathematical sciences publishers

nonprofit scientific publishing

http://msp.org/

(C) 2013 Mathematical Sciences Publishers 


\section{Algebra \& Number Theory}

\section{Volume $7 \quad$ No. $1 \quad 2013$}

Powers of ideals and the cohomology of stalks and fibers of morphisms

MARC CHARDIN

Graphs of Hecke operators

OLIVER LORSCHEID

Group actions of prime order on local normal rings

FrANZ KIRÀLY and WERNER LÜTKEBOHMERT

On the arithmetic and geometry of binary Hamiltonian forms

JOUNI PARKKONEN and FRÉDÉRIC PAULIN

$L$-functions and periods of adjoint motives

MICHAEL HARRIS

Galois module structure of local unit groups

ROMYAR SHARIFI

On the invariant theory for tame tilted algebras

CALIN CHINDRIS

Period functions and cotangent sums

SANDRO BETTIN and BRIAN CONREY 